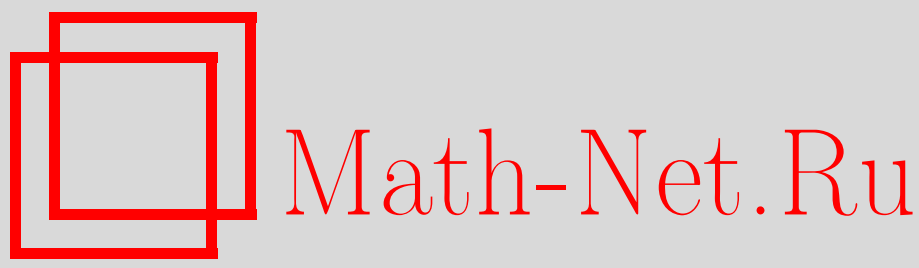

А. А. Рыжков, В. И. Черноусов, О классификации максимальных арифметических подгрупп односвязных групп, Матем. сб., 1997, том 188, номер 9, 127-156

DOI: https://doi.org/10.4213/sm260

Использование Общероссийского математического портала Math-Net.Ru подразумевает, что вы прочитали и согласны с пользовательским соглашением

http://www . mathnet.ru/rus/agreement

Параметры загрузки:

IP: 34.229 .108 .108

26 апреля 2023 г., $13: 31: 26$ 
УДК 512.743

\author{
А. А. Рыжков, В. И. Черноусов
}

\title{
О классификации максимальных арифметических подгрупп односвязных групп
}

\begin{abstract}
Пусть $G \subset \mathrm{GL}_{n}-$ простая односвязная алгебраическая группа, определенная над полем алгебраических чисел $K, T$ - множество всех неархимедовых нормирований $v$ поля $K$. Хорошо известно, что любая максимальная арифметическая подгруппа $\Gamma \subset G$ может быть однозначно восстановлена при помощи набора парахорических подгрупп; более точно, существуют парахорические подгруппы $M_{v} \subset G\left(K_{v}\right), v \in T$, имеющие максимальньй тип и такие, что $\Gamma=\mathrm{N}_{G}(M)$, где $M=G(K) \cap \prod_{v \in T} M_{v}$. Таким образом, возникает естественный вопрос: для каких наборов $\left\{M_{v}\right\}_{v \in T}$, состоящих из парахорических подгрупп $M_{v} \subset G\left(K_{v}\right)$ максимальных типов, определенная вьше подгруппа $\Gamma \subset G$ является максимальной ариффметической подгруппой в $G$. Используя когомологический критерий Рольфса максимальности арифметических подгрупп, мы находим необходимые и достаточные условия максимальности вьшеопределенной арифметической подгруппы $\Gamma \subset G$. Ответ дан в терминах существования элементов поля $K$ с предписанньми свойствами делимости.

Библиография: 23 названия.
\end{abstract}

\section{Введение}

Настоящая работа инспирирована серией работ А. А. Бондаренко [1]-[7], в которых приводится классификация максимальных арифметических подгрупп (всюду далее для краткости МАП) в ортогональных и симплектических группах, определенных над полями алгебраических чисел. Эти работы, на наш взгляд, имеют ряд недостатков. Главньм из них, без сомнения, является ошибочность формулировок основных результатов работ [5], [6], [7], так как приведенные в этих работах необходимые и достаточные условия максимальности арифметической подгрупшы являются лишш необходимыми, но не достаточными; соответствующий контрпример строится в конце статьи.

Далее, способ доказательства, избранньй Бондаренко, заведомо не является оптимальным. Его рассуждения основаны на использовании явных геометрических реализаций исследуемых груп, что, с одной стороны, не позволяет включить в рассмотрение группы исключительных типов, которые, как известно, не имеют хороших геометрических реализаций, а с другой стороны, не является эффективным даже для групп классических типов. Это подтверждается как многочисленным дублированием однотипных рассуждений в упомянутых выше работах, так и тем фактом, что в них не охвачены все формы групп типов $C_{n}, D_{n}$.

Наконец, следует сказать, что при описании МАП наиболее естественньп является получение их классификации с точностью до сопряжения элементами группы $K$-точек односвязных групп $G$ (а не их центральных факторов $G / \mu$, как это 
сделано, например, в [6] для ортогональных групп), так как из сопряженности $\Gamma_{2}=x \Gamma_{1} x^{-1}$ двух МАП $\Gamma_{1}, \Gamma_{2}$ элементом $x$, образ которого лежит в $K$-точках фактора $G / \mu$, не следует их сопряженность элементом из $G(K)$. Забегая вперед, отметим также, что чем меньше центр исследуемой группы $G$, тем меньше технических трудностей приходится преодолевать на пути получения классификации МАП. Например, ответ давно уже известен для групп, являющихся одновременно односвязными и присоединенньми [8], [9] .

В предлагаемой статье приводится классификация МАП простых односвязных групп $G$ всех типов с точностью до сопряжения элементами из $G(K)$. Наши рассуждения не зависят от выбора представления групшы $G$, имеют универсальный характер и базируются на систематическом использовании структурных и арифметических свойств алгебраических групп, определенных над локальными и глобальньми полями. Основными ингредиентами доказательств являются теория Брюа-Титса [10], [11], теорема о сильной аппроксимации [12], принцип Хассе для когомологий односвязных групп [12] и когомологический критерий Рольфса максимальности арифметических подгрупп (см. [13], [14]). Отправной точкой получения явной классификации МАП является для нас локально-глобальный подход, разработанный в 60-х-70-х годах в работах целого ряда математиков (см., например, [8], [13]-[17] и содержащиеся в обзоре В. П. Платонова [9] библиографические замечания). Его суть заключается в следующем.

Пусть $G \subset \mathrm{GL}_{n}$ - абсолютно простая односвязная алгебраическая группа, определенная над полем алгебраических чисел $K$. Обозначим через $\mathscr{O}$ кольцо целых алгебраических чисел поля $K$, через $V$ (соответственно, $V_{\infty}$ и $\left.V_{f}\right)$ - множество всех попарно неэквивалентных (соответственно, архимедовых и неархимедовых) нормирований поля $K$. Пусть также $\mu$ - центр группы $G$ и $\pi: G \rightarrow \bar{G}=G / \mu-$ канонический гомоморфизм в соответствующую присоединенную группу. В дальнейшем будем предполагать, что вешественная часть $\prod_{v \in V_{\infty}} G\left(K_{v}\right)$ группы аделей $G(A)$ некомпактна. Последнее условие естественно, так как оно эквивалентно бесконечности группы целых точек $G(\mathscr{O})$.

Хорошо известно, что любая МАП $\Gamma \subset G$ может быть однозначно восстановлена при помощи набора локальных открытых компактных подгрупп $M_{v} \subseteq G\left(K_{v}\right)$, $v \in V_{f}$, выбранных специальным образом. Более точно, рассмотрим образ $\pi(\Gamma) \subseteq \bar{G}$. Поскольку он лежит в $\bar{G}(K)$, то можно взять замькание $\pi(\Gamma)_{v}=$ $\overline{\pi(\Gamma)} \subseteq \bar{G}\left(K_{v}\right)$ в $v$-адической топологии. Это замыкание является открытой компактной (но, вообше говоря, немаксимальной) подгруппой. Обозначим через $\bar{M}_{v}$ произвольную максимальную компактную подгруппу, содержашую $\pi(\Gamma)_{v}$, и положим $M_{v}=\pi^{-1}\left(\bar{M}_{v}\right) \cap G\left(K_{v}\right)$. Согласно нашему построению группа Г лежит в арифметической подгрупше $N_{G}(M)$, где $M=G(K) \cap \prod_{v \in V_{f}} M_{v}$, и значит, в силу максимальности обязана с ней совпадать.

Естественным образом возникает обратный вопрос, который приобрел в литературе название проблемы максимальности: найти необходимые и достаточные условия на множество открытых компактных подгрупп $\left\{M_{v}\right\}_{v \in V_{f}}, M_{v} \subseteq G\left(K_{v}\right)$, которые гарантируют максимальность арифметической подгруппы $\Gamma=N_{G}(M)$, где, как и вьше, $M=G(K) \cap \prod_{v \in V_{f}} M_{v}$.

Легко установить, что для максимальности $Г$ необходимо, чтобы локальные 
подгрупшы $M_{v}$ были парахорическими подгруппами и имели максимальный тип (см. определение в $\S 1$ ), причем $M_{v}=G\left(\mathscr{O}_{v}\right)$ для почти всех $v \in V_{f}$. Примеры показывают, что в обшем случае эти условия не являются достаточными. Чтобы получить критерий максимальности $\Gamma$ в классе $K$-разложимых груп $G$, Рольфс в работе [13] сузил класс парахорических подгрупп максимального типа, введя понятие подгруппы $\mathscr{O}$-максимального типа, и доказал, что если $G$ разложима над $K$, то Г является максимальной тогда и только тогда, когда набор $\left\{M_{v}\right\}_{v \in V_{f}}$ имеет $\mathscr{O}$-максимальный тип. Отметим, что для $K$-разложимых групп $G$, определенных над одноклассньм полем $K$, понятия максимальности и $\mathscr{O}$-максимальности совпадают, и поэтому отсюда в качестве частных случаев при $G=\mathrm{SL}$, Sp следуют результаты работ Аллана [16], [17], а при $K=\mathbb{Q}$ - результаты В.П. Платонова [8].

Анализ доказательства Рольфса показьвает, что его критерий максимальности $\Gamma$ справедлив для всех групा $G$, а не только для $K$-разложимых. Поскольку дополнительно в указанном выше способе построения МАП можно ограничиться стандартными парахорическими подгруппами, каноническое описание которых доставляет теория Брюа-Титса, то фактически на критерий Рольфса уже можно смотреть как на классификацию МАП с точностью до сопряжения элементами из $G(K)$ (см. формулировку теоремы 2.6). Единственный недостаток такой классификации состоит в том, что она дается в когомологических терминах (см. определение $\mathscr{O}$-максимального типа в $\S 2)$, что не всегда удобно для применений. В настоящей работе, опираясь на критерий Рольфса, мы находим необходимые и достаточные условия максимальности Г в терминах существования элементов в исходном поле $K$ с предписанными свойствами делимости и, как следствие, приводим классификацию МАП простых односвязных групп всех типов в том виде, к которому стремился А. А. Бондаренко в своих работах [1]-[7]. Тем самым, нашу статью можно рассматривать как естественное дополнение к [13].

Из-за громоздкости мы не будем приводить во введении формулировки основных результатов, а в заключение остановимся лишш на структуре работы. Первый параграф посвяшен арифметическим и парахорическим подгруппам. В нем собраны необходимые в дальнейшем свойства этих подгрупп. Все они хорошо известны. Там, где не требуются большие усилия, приводятся полные доказательства; в остальных местах даются точные ссылки. В 22 излагается критерий Рольфса для произвольной односвязной группы $G$. В общем случае он впервые был сформулирован без доказательства в работе Рольфса и Маргулиса [14]. Поскольку с тех пор доказательство не было опубликовано, то мы решили для полноты привести его; при изложении доказательства мы полностью следуем рассуждениям Рольфса из [13]. В $\S 3$ дается явное описание множества $\operatorname{Im}\left(\rho: \bar{G}(K) \rightarrow H^{1}(K, \mu)\right)$, где $\rho$-канонический гомоморфизм, индуцированный точной последовательностью $1 \rightarrow \mu \rightarrow G \rightarrow \bar{G} \rightarrow 1$. Результаты этого параграффа и критерий Рольфса применяются в последуюших параграфах для получения классификации МАП групп типов $G_{2}, F_{4}, E_{8}, E_{7}, E_{6}, B_{n}, C_{n}$. Наиболее сложная в техническом плане классификация МАП групп типов $A_{n}, D_{n}$ будет дана во второй части статьи. Кроме того, здесь же будет построен пример ортогональной групшы $\mathrm{SO}(f)$ и ее арифметической подгруппы $\Gamma$, которая удовлетворяет необходимым и достаточным условиям мак- 
симальности, сфформулированным в работе А. А. Бондаренко [5], но которая, тем не менее, не является максимальной.

Всюду ниже через $G$ (если не оговорено противное) обозначается односвязная абсолютно простая алгебраическая группа, определенная над полем алгебраических чисел $K$. Кроме того, мы фиксируем ее $K$-реализацию $G \subset \mathrm{GL}_{n}$.

Работа над настоящей статьей была начата в 1995 г. во время визита второго автора в Университет Билефельда в качестве гостя SFB 343 "Diskrete Strukturen in der Mathematik" и закончена в 1996 г. во время визита в тот же Университет в качестве стипендиата фонда А. Гумбольдта. Он благодарит фонд Гумбольдта и SFB 343 за поддержку, а Университет Билефельда за гостеприимство.

\section{§1. Арифметические и парахорические подгруппы и их свойства}

1.1. Напомним, что подгруппа $H \leqslant G(\bar{K})$ называется арифметической, если она соизмерима с группой целых точек $G(\mathscr{O})$, т.е. пересечение $H \cap G(\mathscr{O})$ имеет конечный индекс как в $H$, так и в $G(\mathscr{O})$, где $G(\mathscr{O})=G(K) \cap \mathrm{GL}_{n}(\mathscr{O})$. Без ограничения обшности мы можем считать [8], что $G \subset \mathrm{GL}_{n}(\mathbb{C})$ реализована таким образом, что $G\left(\mathscr{O}_{v}\right)$ - максимальная открытая компактная подгруппа групшы $G\left(K_{v}\right)$ для всех $v \in V_{f}$. Всюду в этом параграфе через $H$ будет обозначаться арифметическая подгруппа произвольной полупростой (не обязательно односвязной) групшы $G$. Отметим, что мы не требуем, чтобы $H$ лежала в $G(K)$.

ПреДЛОЖЕнИЕ. Пусть $f: G \rightarrow G^{\prime}-$ центральная $K$-изогения. Тогда подгруппа $H \leqslant G(\bar{K})$ является арифметической (соответственно, максимальной арифметической) тогда и только тогда, когда образ $f(H)$ является арифметической (соответственно, максимальной арифметической).

ДокАЗАТЕЛЬСтво. См. [18] или [12, гл. 5, §4, теорема 9].

1.2. ПРЕДЛОЖЕНИЕ. Если иентр и группь $G$ тривиален, то $H \leqslant G(K)$.

ДокаЗАтельство. По условию $[H: H \cap G(\mathscr{O})]<\infty$. Поэтому найдется нормальный делитель конечного индекса $H_{0} \leqslant H$, лежащий в $G(\mathscr{O})$. Рассмотрим групповую $K$-алгебру $K\left[H_{0}\right]$. Так как арифметические подгрупшы плотны в топологии Зариского [18], то $K\left[H_{0}\right] \otimes_{K} \bar{K}=\bar{K}\left[H_{0}\right]=\bar{K}[G]$. Обозначим через $\lambda: G \rightarrow \bar{K}[G]$ соответствуюший присоединенный гомоморфизм. Тогда, с одной стороны, $\lambda-K$-изоморфизм между $G$ и $\lambda(G)$, ибо центр $G$ тривиален, а с другой стороны, образ $\lambda(H)$ по построению лежит в $K$-точках группы $\lambda(G)$. Это означает, что $H \leqslant G(K)$, что и требовалось доказать.

1.3. ПРЕДЛОЖЕНИЕ (свойства компактных подгрупп группы $\left.G\left(K_{v}\right)\right)$.

а) Любая открытая компактная подгруппа группь $G\left(K_{v}\right), v \in V_{f}$, содержится в конечном числе компактных подгрупп.

б) Любая компактная подгруппа группь $G\left(K_{v}\right)$ содерэсится в некоторой максимальной компактной подгруппе.

ДоказАТельство. См., например, [12, гл. $3, \S 3]$. 
1.4. ПрЕДЛОЖЕНИЕ. Пусть $H \leqslant G(K)$. Тогда замыкание $H_{v}$ группы $H$ в $v$-адической топологии является открытой компактной подгруппой группы $G\left(K_{v}\right)$ для всех $v \in V_{f}$.

ДокаЗАТЕЛЬСтво. Очевидно, что достаточно проверить это утверждение хотя бы для одной арифметической подгруппы $\Gamma \leqslant G(K)$. Если группа $G$ односвязна, то в качестве $Г$ можно взять группу целых точек $G(\mathscr{O})$, так как из сильной аппроксимационной теоремы вытекает, что $G(\mathscr{O})_{v}=\overline{G(\mathscr{O})}=G\left(\mathscr{O}_{v}\right)$ и $G\left(\mathscr{O}_{v}\right)$ - открытая компактная подгруппа группы $G\left(K_{v}\right)$. В общем случае, пусть $\widetilde{G}$ - односвязная накрывающая и $\pi: \widetilde{G} \rightarrow G$-канонический гомоморфизм. Согласно предложению 1.1 подгруппа $\Gamma=\pi(\widetilde{G}(\mathscr{O}))$ является арифметической. Тогда из открытости морфизма $\pi$ в $v$-адической топологии следует, что $\pi\left(\widetilde{G}\left(\mathscr{O}_{v}\right)\right)$ - открытая компактная подгруппа в $G\left(K_{v}\right)$, и по построению Г плотна в ней. Предложение доказано.

1.5. ПрЕДЛОЖЕНИЕ. Пусть $H \leqslant G(K)$. Тогда $N_{G(\bar{K})}(H)$ является арифметической подгруппой.

ДоказАтЕльство. См., например, [12, гл. 3, §3, предл. 15], содержашее доказательство в локальном случае (отметим, что оно работает и в глобальном случае), или же [20], где рассматривается более обший случай решеток.

1.6. ПрЕДЛОЖЕНИЕ. Пусть $G$ - односвязная группа. Если $H \leqslant G(K)$, mo для почти всех $v \in V_{f}$ подгруппа $H_{v}$ совпадает $c G\left(\mathscr{O}_{v}\right)$.

ДоКАЗАТЕЛЬСТВо. Без ограничения общности можно считать, что $H \leqslant G(\mathscr{O})$. Пусть $v_{1}, \ldots, v_{n}-$ произвольные нормирования из $V_{f}$. Из сильной аппроксимационной теоремы и предложения 1.4 следует, что

$$
G\left(\mathscr{O}_{v_{1}}\right) \times \cdots \times G\left(\mathscr{O}_{v_{n}}\right)=i(G(\mathscr{O})) \cdot\left(H_{v_{1}} \times \cdots \times H_{v_{n}}\right),
$$

где $i: G(\mathscr{O}) \rightarrow G\left(\mathscr{O}_{v_{1}}\right) \times \cdots \times G\left(\mathscr{O}_{v_{n}}\right)$ - диагональное вложение. Тогда имеем:

$$
\begin{aligned}
\prod_{i=1}^{n} G\left(\mathscr{O}_{v_{i}}\right) / \prod_{i=1}^{n} H_{v_{i}} & =\left(i(G(\mathscr{O})) \cdot \prod_{i=1}^{n} H_{v_{i}}\right) / \prod_{i=1}^{n} H_{v_{i}} \\
& \simeq i(G(\mathscr{O})) /\left(i(G(\mathscr{O})) \cap \prod_{i=1}^{n} H_{v_{i}}\right) .
\end{aligned}
$$

Но $i(H) \leqslant i(G(\mathscr{O})) \cap \prod_{i=1}^{n} H_{v_{i}}$, поэтому

$$
\prod_{i=1}^{n}\left[G\left(\mathscr{O}_{v_{i}}\right): H_{v_{i}}\right]=\left[i(G(\mathscr{O})):\left(i(G(\mathscr{O})) \cap \prod_{i=1}^{n} H_{v_{i}}\right)\right] \leqslant[G(\mathscr{O}): H]<\infty,
$$

откуда следует, что имеется лишь конечное число нормирований $v \in V_{f}$, для которых $H_{v} \neq G\left(\mathscr{O}_{v}\right)$. Предложение доказано.

1.7. Пусть $v \in V_{f}$ и $G$ - односвязна. Напомним [10], [11], что на группе $G\left(K_{v}\right)$ имеется структура системы Титса афффинного типа $\left(G\left(K_{v}\right), B_{v}, N_{v}, \Delta_{v}\right)$ с группой Вейля $W_{v}$. 
ОПРЕДЕЛЕНИЕ. Подгруппа $P_{v}<G\left(K_{v}\right)$ называется подгруппой Ивахори, если она сопряжена в $G\left(K_{v}\right)$ подгруппе $B_{v} . P_{v}$ называется парахорической nодгруnnой, если она содержит подгруппу Ивахори.

Любая парахорическая подгруппа $P_{v}$ сопряжена в $G\left(K_{v}\right)$ единственной $c m a н-$ дартной парахорической подгруппе $P_{X_{v}}$ вида $P_{X_{v}}=B_{v} W_{X_{v}} B_{v}$, где $X_{v} \subset \Delta_{v}$ - некоторое подмножество [19]. Это подмножество $X_{v}$ называется типом парахорической подгруппы $P_{v}$ и обозначается через $\tau\left(P_{v}\right)$. Если $X_{v}=\Delta \backslash\left\{\alpha_{0}\right\}$, то $P_{X_{v}}$ совпадает с $G\left(\mathscr{O}_{v}\right)[11]$.

1.8. Группа $\bar{G}\left(K_{v}\right)$ естественньм образом действует на множестве парахорических подгрупп. А именно, если $g \in \bar{G}\left(K_{v}\right)$ и $P_{v} \leqslant G\left(K_{v}\right)$ - парахорическая подгруппа, то по определению $g \cdot\left(P_{v}\right)=\widetilde{g} P_{v} \widetilde{g}^{-1}$, где $\widetilde{g} \in \pi^{-1}(g)$ - произвольньй прообраз. Легко проверить, что это действие определено корректно. Так как классы сопряженных в $G\left(K_{v}\right)$ парахорических подгрупп однозначно определяются своими типами, то в результате получаем каноническое действие группы $\bar{G}\left(K_{v}\right)$ на соответствуюшем графе Кокстера $\Delta_{v}$. Обозначим это действие через $\xi_{v}: \bar{G}\left(K_{v}\right) \rightarrow$ Aut $\Delta_{v}$. Очевидно, что $\pi\left(G\left(K_{v}\right)\right) \subseteq \operatorname{Ker} \xi_{v}$.

Зададим также действие $H^{1}\left(K_{v}, \mu\right)$ на $\Delta_{v}$. Из тривиальности $H^{1}\left(K_{v}, G\right)[12]$ следует, что имеется точная последовательность

$$
1 \rightarrow \mu\left(K_{v}\right) \rightarrow G\left(K_{v}\right) \stackrel{\pi}{\longrightarrow} \bar{G}\left(K_{v}\right) \stackrel{\rho}{\rightarrow} H^{1}\left(K_{v}, \mu\right) \rightarrow 1 .
$$

Тогда для любого элемента $\gamma \in H^{1}\left(K_{v}, \mu\right)$ слой $\rho^{-1}(\gamma)$ непуст, и поэтому $\gamma$ можно поставить в соответствие автоморфизм $\xi_{v}(g)$, где $g \in \rho^{-1}(\gamma)$ - произвольный прообраз. Этот автоморфизм не зависит от выбора представителя $g$, ибо $\pi\left(G\left(K_{v}\right)\right) \subseteq$ $\operatorname{Ker} \xi_{v}$, и тем самым в результате получаем гомоморфизм $H^{1}\left(K_{v}, \mu\right) \rightarrow$ Aut $\Delta_{v}$, которьй будем обозначать той же буквой $\xi_{v}$. Итак, имеется коммутативная диаграмма

$$
\begin{gathered}
\bar{G}\left(K_{v}\right) \stackrel{\xi_{v}}{\longrightarrow} \text { Aut } \Delta_{v} \\
\rho \searrow \xi_{v} \\
H^{1}\left(K_{v}, \mu\right) .
\end{gathered}
$$

Положим $\Xi_{v}=\operatorname{Im} \xi_{v}$.

1.9. ОПРЕДЕЛЕНИЕ. Тип $X_{v} \subseteq \Delta_{v}$ называется максимальным, если дополнение $\Delta_{v} \backslash X_{v}$ является орбитой некоторой подгруппы группы $\Xi_{v}$.

Пример. Если $\Delta_{v} \backslash X_{v}$ - одна вершина, то тип $X_{v}$ максимален, так как любая вершина является орбитой единичной подгруппы.

ПРЕДЛОЖЕНИЕ. Пусть $M_{v} \leqslant \bar{G}\left(K_{v}\right)$ u $P_{v}=G\left(K_{v}\right) \cap \pi^{-1}\left(M_{v}\right)$. Тогда $M_{v}$ является максимальной компактной подгруппой группы $\bar{G}\left(K_{v}\right)$ тогда и только тогда, когда $P_{v}$ - парахорическая подгруппа максимального типа.

ДоказАТеЛЬСтво. См. [10]. 
1.10. Лемма. Пусть $\Gamma \leqslant G$ - арифметическая подгруппа и $P=\Gamma \cap G(K)$. Тогда $\Gamma \leqslant N_{G(\bar{K})}(P)$.

ДокаЗАтельство. Достаточно установить, что $[\Gamma, \Gamma] \subset P$. Пусть $\gamma_{1}, \gamma_{2} \in \Gamma$ и $\sigma \in \operatorname{Gal}(\bar{K} / K)$. Тогда в силу предложения 1.2 элемент $\gamma_{i}^{-1} \sigma\left(\gamma_{i}\right)$ лежит в центре групшы $G$, откуда $\sigma\left(\gamma_{1} \gamma_{2} \gamma_{1}^{-1} \gamma_{2}^{-1}\right)=\gamma_{1} \gamma_{2} \gamma_{1}^{-1} \gamma_{2}^{-1}$ и, следовательно, $[\Gamma, \Gamma] \subset G(K)$. Лемма доказана.

\section{§2. Критерий Рольфса максимальности арифметической подгруппы}

2.1. Начиная с этого места, через $G$ будет обозначаться простая односвязная алгебраическая $K$-группа.

ОпРЕДЕЛЕНИЕ. Арифметическая подгруппа $H \leqslant G(K)$ называется парахорической, если для любого $v \in V_{f}$ замыкание $H_{v}=\bar{H}$ является парахорической подгруппой группы $G\left(K_{v}\right)$ и $H=G(K) \cap \prod_{v \in V_{f}} H_{v}$. Если $X_{v}=\tau\left(H_{v}\right)$ есть тип $H_{v}$, то семейство $X=\left\{X_{v}\right\}_{v \in V_{f}}$ называется типом $H$ и обозначается $\tau(H)$.

Отметим, что в силу предложения $1.6 H_{v}=G\left(\mathscr{O}_{v}\right)$ для почти всех $v \in V_{f}$. Следовательно, если $H$ - парахорическая подгруппа и $X$ - ее тип, то для почти всех $v \in V_{f}$ справедливо равенство $X_{v}=\Delta_{v} \backslash\left\{\alpha_{0}\right\}$.

2.2. ОПРеДЕлЕнИЕ. Семейство $X=\left\{X_{v}\right\}$, где $X_{v} \subseteq \Delta_{v}$, называется типом, если $X_{v}=\Delta_{v} \backslash\left\{\alpha_{0}\right\}$ для почти всех $v \in V_{f}$.

Если $X=\left\{X_{v}\right\}$ - тип, то положим $P_{X}=G(K) \cap \prod_{v \in V_{f}} P_{X_{v}}$. Очевидно, что $P_{X}$ является арифметической подгруппой и ее замыкание $\overline{P_{X}}$ в $v$-адической топологии совпадает с $P_{X_{v}}$. Это означает, что $P_{X}$ - парахорическая подгруппа типа $X$. В дальнейшем будем назьвать ее стандартной парахорической подгруппой.

ПРЕДЛОЖЕНИЕ. Любая парахорическая подгруппа $P \leqslant G(K)$ сопряжена $в$ $G(K)$ ровно с одной стандартной парахорической подгруппой.

ДоказАтельство. Пусть $X=\left\{X_{v}\right\}$ - тип подгруппы $P$ и рассмотрим соответствующую стандартную парахорическую подгруппу $P_{X}$. По построению подгруппы $P_{v}$ и $P_{X_{v}}$ сопряжены в $G\left(K_{v}\right)$. Введем множество

$$
R=\left\{g \in G\left(A_{f}\right) \mid g\left(\prod_{v \in V_{f}} P_{X_{v}}\right) g^{-1}=\prod_{v \in V_{f}} P_{v}\right\} .
$$

Применяя предложение 1.6, получаем, что множество $T=\left\{v \in V_{f} \mid P_{X_{v}} \neq P_{v}\right\}$ конечно, откуда следует, что $R$ - непусто. Кроме того $R$, очевидно, является открытым подмножеством, ибо если $g=\left(g_{v}\right) \in R$, то и

$$
\prod_{v \in T} g_{v} P_{X_{v}} \times \prod_{v \notin T} P_{X_{v}} \subset R
$$

Тогда из сильной аппроксимационной теоремы следует, что пересечение $G(K) \cap R$ плотно в $R$ и, в частности, не является пустым. Для любого элемента $r \in R \cap G(K)$ 
имеем:

$$
\begin{aligned}
r P_{X} r^{-1} & =r\left(G(K) \cap \prod_{v \in V_{f}} P_{X_{v}}\right) r^{-1} \\
& =G(K) \cap r\left(\prod_{v \in V_{f}} P_{X_{v}}\right) r^{-1}=G(K) \cap \prod_{v \in V_{f}} P_{v}=P
\end{aligned}
$$

т.е. $P$ сопряжена со стандартной парахорической подгруппой $P_{X}$. Утверждение о единственности $X$ очевидно. Предложение доказано.

2.3. Введем следующие обозначения. Если $X=\left\{X_{v}\right\}_{v \in V_{f}}$ - тип, то положим $\Xi_{X_{v}}-$ стабилизатор подмножества $X_{v}$ в группе $\Xi_{v}$ и $H^{1}\left(K_{v}, \mu\right)_{X_{v}}=\xi_{v}^{-1}\left(\Xi_{X_{v}}\right)$, где $\xi_{v}$ - гомоморфизм, построенньй в пункте 1.8. Пусть также $\lambda: H^{1}(K, \mu) \rightarrow$ $\prod_{v \in V_{f}} H^{1}\left(K_{v}, \mu\right)$ - канонический гомоморфизм. Положим

$$
H^{1}(K, \mu)_{X}=\lambda^{-1}\left(\prod_{v \in V_{f}} H^{1}\left(K_{v}, \mu\right)_{X_{v}}\right) \cap \operatorname{Im} \rho
$$

где $\rho: \bar{G}(K) \rightarrow H^{1}(K, \mu)$ - гомоморфизм, индуцированный точной последовательностью $1 \rightarrow \mu \rightarrow G \rightarrow \bar{G} \rightarrow 1$.

Лемма. Пусть $P \leqslant G(K)$ - парахорическая подгруппа muпа $X=\left\{X_{v}\right\}_{v \in V_{f}}$ u $\Gamma=N_{G(\bar{K})}(P)$. Тогда для любого әлемента $\gamma \in \Gamma$ класс коцикла $\left(a_{\sigma}\right)=$ $\left(\gamma^{-1} \sigma(\gamma)\right)_{\sigma \in \operatorname{Gal}(\bar{K} / K)}$ лехит в $H^{1}(K, \mu)_{X}$.

ДокАЗАТЕЛьство непосредственно вытекает из предложений 1.2, 1.5. Действительно, поскольку $\Gamma$ - арифметическая подгруппа, то согласно $1.2 \pi(\Gamma) \leqslant \bar{G}(K)$, откуда $\left[\left(a_{\sigma}\right)\right] \in H^{1}(K, \mu)$. Кроме того, по построению

$$
\left[\left(a_{\sigma}\right)\right] \in \operatorname{Im}\left(\rho: \bar{G}(K) \rightarrow H^{1}(K, \mu)\right)
$$

ибо $\left[\left(a_{\sigma}\right)\right]=\rho(\pi(\gamma))$. Наконец, при сопряжении $\gamma$ оставляет на месте $P$, а значит, и ее замыкание $P_{v}$. В частности, это означает, что сопрягая элементом $\gamma$ парахорическую подгруппу $P_{v}$, мы не меняем ее тип $X_{v}$, откуда $\operatorname{Res}_{K_{v}}\left(\left[\left(a_{\sigma}\right)\right]\right) \in \xi_{v}^{-1}\left(\Xi_{X_{v}}\right)$ для всех $v \in V_{f}$, что и требовалось доказать. Лемма доказана.

2.4. Согласно предыдущей лемме имеется канонический гомоморфизм

$$
\delta_{X}: \Gamma \rightarrow H^{1}(K, \mu)_{X}
$$

ПРЕДЛОЖЕНИЕ. Гомоморфизм $\delta_{X}$ сюрвективен. 
ДокАЗАТЕЛЬСТво. Пусть $c \in H^{1}(K, \mu)_{X}$ - произвольный элемент и $\left(a_{\sigma}\right)-$ представляющий его коцикл. Поскольку $c \in \operatorname{Im} \rho$, то найдется такой $g \in \bar{G}(K)$, что $\rho(g)=c$ или же $\widetilde{g}^{-1} \sigma(\widetilde{g})=a_{\sigma}$ для всех $\sigma \in \operatorname{Gal}(\bar{K} / K)$, где $\widetilde{g} \in \pi^{-1}(g)-$ произвольный прообраз элемента $g$. Из условия $c \in H^{1}(K, \mu)_{X}$ вытекает также, что для любого $v \in V_{f}$ подгруппа $\widetilde{g} P_{v} \widetilde{g}^{-1}$ является парахорической подгруппой того же типа $X_{v}$, откуда $\widetilde{g} P_{v} \widetilde{g}^{-1}=h_{v} P_{v} h_{v}{ }^{-1}$ для некоторого $h_{v} \in G\left(K_{v}\right)$. Тогда, применяя сильную аппроксимационную теорему, мы можем найти один элемент $h \in G(K)$, для которого справедливы равенства

$$
\widetilde{g} P_{v} \widetilde{g}^{-1}=h_{v} P_{v} g_{v}^{-1}=h P_{v} h^{-1}
$$

для всех $v \in V_{f}$. Отсюда следует, что $h^{-1} \widetilde{g} \in N_{G(\bar{K})}(P)$ и

$$
\left(h^{-1} \widetilde{g}\right)^{-1} \sigma\left(h^{-1} \widetilde{g}\right)=\widetilde{g}^{-1}\left(h \sigma\left(h^{-1}\right)\right) \sigma(\widetilde{g})=\widetilde{g}^{-1} \sigma(\widetilde{g})=a_{\sigma},
$$

т.е. $\delta_{X}\left(h^{-1} \widetilde{g}\right)=c$. Предложение доказано.

2.5. ОПРЕДЕЛЕНИЕ. 1) Тип $X=\left\{X_{v}\right\}_{v \in V_{f}}$ называется максимальныцм, если $X_{v}$ - максимальный тип для всех $v \in V_{f}$.

2) Максимальньй тип $X$ назьвается $\mathscr{O}$-максимальным, если для всех максимальных типов $X^{\prime}$, строго содержащих $X$, включение $H^{1}(K, \mu)_{X^{\prime}} \subset H^{1}(K, \mu)_{X}$ также строгое.

Для типа $X=\left\{X_{v}\right\}_{v \in V_{f}}$ положим $\Gamma_{X}=N_{G(\bar{K})}\left(P_{X}\right)$.

2.6. ТЕорема (критерий максимальности Рольфса).

1) Каждая максимальная арифметическая подгруппа сопряжена әлементом из $G(K)$ ровно с одной подгруппой $\Gamma_{X}$.

2) $\Gamma_{X}$ является максимальной арифметической подгруппой тогда и только тогда, когда $X-\mathscr{O}$-максимальный тип.

ДокАЗАТЕЛЬСТво. 1) Пусть $Г$ - максимальная арифметическая подгруппа. Ее образ $\bar{\Gamma}=\pi(\Gamma)$ в силу предложений $1.1,1.2$ является максимальной арифметической подгруппой в $\bar{G}(K)$, и поэтому замькание $\bar{\Gamma}_{v}$ подгруппы $\bar{\Gamma}$ в $\bar{G}\left(K_{v}\right)$ есть открытая компактная подгруппа (см. 1.4). Согласно предложению $1.3 \bar{\Gamma}_{v}$ содержится в некоторой максимальной компактной подгруппе $M_{v} \leqslant \bar{G}\left(K_{v}\right)$. Рассмотрим подг руппу $P_{v}=\pi^{-1}\left(M_{v}\right) \cap G\left(K_{v}\right)$. Применяя предложение 1.9 , имеем: $P_{v}-$ парахорическая подгруппа максимального типа, и по построению для почти всех $v \in V_{f}$ справедливо равенство $P_{v}=G\left(\mathscr{O}_{v}\right)$. Тем самым, арифметическая подгруппа $P=G(K) \cap \prod_{v \in V_{f}} P_{v}$ также является парахорической и имеет максимальный тип.

Из рассуждений в лемме 1.10 следует, что $\Gamma$ нормализует как группу $G(K)$, так и подг руппу $P_{v}$ для всех $v \in V_{f}$, ибо по построению $\Gamma \subseteq \pi^{-1}\left(M_{v}\right)$. Отсюда следует, что $Г$ нормализует также $P$, т.е. $\Gamma \subseteq N_{G(\bar{K})}(P)$, и поскольку $N_{G(\bar{K})}(P)$ - арифметическая подгруппа, а Г по условию максимальна, то обязательно $\Gamma=N_{G(\bar{K})}(P)$. Остается заметить, что если $X=\tau(P)$ - тип парахорической подгруппы $P$, то из предложения 2.2 следует, что подгруппа $P$ и стандартная парахорическая подгруппа $P_{X}$ сопряжены в $G(K)$, и тем самым $\Gamma$ и $\Gamma_{X}$ также сопряжены при помоши 
элемента из $G(K)$. Что касается утверждения о единственности $X$, то оно очевидно.

2) Пусть вначале $\Gamma_{X}$ - максимальная арифметическая подгруппа. Из доказательства первой части теоремы следует, что ее тип является максимальньм, и остается доказать, что он $\mathscr{O}$-максимален.

Предположим, что это не так. Тогда найдется максимальный тип $X^{\prime}$, который строго содержит $X$, и такой, что $H^{1}(K, \mu)_{X}=H^{1}(K, \mu)_{X^{\prime}}$.

Рассмотрим диаграмму:

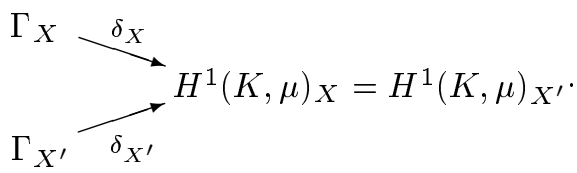

Из способа построения морфизмов $\delta_{X}$ и $\delta_{X^{\prime}}$ и их сюръективности вытекает, что для любого $a \in \Gamma_{X}$ сушествуют $b \in \Gamma_{X^{\prime}}$ и $g \in G(K)$ такие, что $a=b g$. Тогда подгруппа $a^{-1} P_{X^{\prime}} a$ является парахорической типа $X^{\prime}$, ибо

$$
a^{-1} P_{X^{\prime}} a=g^{-1} b^{-1} P_{X^{\prime}} b g=g^{-1} P_{X^{\prime}} g \leqslant G(K)
$$

Кроме того, условие $X \subset X^{\prime}$ влечет включение $P_{X} \subset P_{X^{\prime}}$, и значит,

$$
P_{X}=a^{-1} P_{X} a \subset a^{-1} P_{X^{\prime}} a=g^{-1} P_{X^{\prime}} g .
$$

Таким образом, парахорическая подгруппа $g^{-1} P_{X^{\prime}} g$ типа $X^{\prime}$ содержит стандартную парахорическую подгруппу $P_{X}$. Отсюда сразу следует, что она также является стандартной [19], т.е. $g^{-1} P_{X^{\prime}} g=P_{X^{\prime}}$; в частности, $g \in P_{X^{\prime}}$, ибо все парахорические подгруппы совпадают со своим нормализатором [19]. Последнее означает, что $a=b g \in \Gamma_{X^{\prime}}$, откуда $\Gamma_{X} \subseteq \Gamma_{X^{\prime}}$, и следовательно, в силу максимальности имеем $\Gamma_{X}=\Gamma_{X^{\prime}}$. С другой стороны, $\Gamma_{X} \cap G(K)=P_{X}, \Gamma_{X^{\prime}} \cap G(K)=P_{X^{\prime}}$ и $P_{X} \neq P_{X^{\prime}}$, ибо включение $X \subset X^{\prime}$ - строгое, т.е. $\Gamma_{X} \neq \Gamma_{X^{\prime}}$ - противоречие, доказываюшее $\mathscr{O}$-максимальность типа $X$.

Обратно, пусть $X-\mathscr{O}$-максимальный тип. Предположим, что $\Gamma_{X}$ не является максимальной, и пусть $\Gamma^{\prime}$ - максимальная арифметическая подгруппа, содержащая $\Gamma_{X}$ (отметим, что такая подгруппа всегда сушествует, так как любая арифметическая подгруппа содержится лишп в конечном числе арифметических подгрупп; доказательство можно найти, например, в [12]). Из утверждения первой части теоремы следует, что $\Gamma^{\prime}=N_{G(\bar{K})}\left(P_{X^{\prime}}\right)$ для некоторого типа $X^{\prime}$. Тогда $\Gamma^{\prime} \cap G(K)=P_{X^{\prime}} \supsetneqq \Gamma_{X} \cap G(K)=P_{X}$, откуда $X^{\prime} \supsetneqq X$.

Далее, по построению $\Gamma_{X} \subset \Gamma^{\prime}=\Gamma_{X^{\prime}}$. Поэтому

$$
\delta_{X}\left(\Gamma_{X}\right)=H^{1}(K, \mu)_{X} \subset \delta_{X^{\prime}}\left(\Gamma_{X^{\prime}}\right)=H^{1}(K, \mu)_{X^{\prime}}
$$

С другой стороны, из включения $X \subset X^{\prime}$ всегда следует включение $H^{1}(K, \mu)_{X^{\prime}} \subset$ $H^{1}(K, \mu)_{X}$, и поэтому справедливо равенство $H^{1}(K, \mu)_{X^{\prime}}=H^{1}(K, \mu)_{X}$, что противоречит условию $\mathscr{O}$-максимальности типа $X$. Теорема полностью доказана. 
§3. Описание $\operatorname{Im}\left(\rho: \bar{G}(K) \rightarrow H^{1}(K, \mu)\right)$

3.1. Точная последовательность $1 \rightarrow \mu \rightarrow G \rightarrow \bar{G} \rightarrow 1$ индуцирует точную последовательность когомологий, из которой следует, что

$$
\operatorname{Im}\left(\rho: \bar{G}(K) \rightarrow H^{1}(K, \mu)\right)=\operatorname{Ker}\left(H^{1}(K, \mu) \rightarrow H^{1}(K, G)\right) .
$$

Поскольку для когомологий односвязных алгебраических групп справедлив принцип Хассе, то коцикл $\left(a_{\sigma}\right) \in Z^{1}(K, \mu)$ тривиален в $H^{1}(K, G)$ тогда и только тогда, когда для всех архимедовых нормирований $v$ поля $K$ он тривиален в $H^{1}\left(K_{v}, G\right)$. Поэтому имеем:

$$
\operatorname{Im}\left(\rho: \bar{G}(K) \rightarrow H^{1}(K, \mu)\right)=\operatorname{Ker}\left(H^{1}(K, \mu) \rightarrow \prod_{v \in V_{\infty}} H^{1}\left(K_{v}, G\right)\right) .
$$

Для описания множества, стояшего в правой части приведенного выше равенства, мы воспользуемся явньпи вычислениями когомологий центров односвязных групп, приведенными в $[12$, с. 366$]$. Всюду в дальнейшем через $\mu_{n}$ будем обозначать $\operatorname{Gal}(\bar{K} / K)$-модуль корней из единицы степени $n$, через $L$ - минимальное расширение поля $K$, над которым группа $G$ имеет внутренний тип, через $\mathbf{R}_{L / K}-$ функтор ограничения поля, через $\mathbf{R}_{L / K}^{(1)}$ - ядро соответствующего норменного отображения, а через $A$ - описываемое множество.

3.2. Далее нам понадобятся несколько стандартных фактов о строении простых односвязных $K$-изотропных групп $G$. А именно, каждой такой группе можно поставить в соответствие геометрическую картинку, называемую $K$-индексом Титса [21]: она состоит из соответствуюшего графа Дьнкина, у которого часть вершин обведена кружками.

Обозначим через $S$ - максимальный $K$-разложимый тор в $G$. Его централизатор $Z_{G}(S)$ является почти прямьм произведением своего центрального тора $S_{1}$ и полупростой $K$-анизотропной группы $Z_{G}(S)^{(1)}=\left[Z_{G}(S), Z_{G}(S)\right]$, называемой полупростым анизотропным ядром группы $G$. Если $G$ есть группа внутреннего типа, то торы $S$ и $S_{1}$ всегда совпадают; в частности, в этом случае $H^{1}\left(K, S_{1}\right)=1$.

Нам понадобится также явное описание тора $S$ над замкнутым полем $\bar{K}$. Чтобы получить его, рассмотрим произвольный максимальный тор $T$ группы $G$, определенный над $K$ и содержащий $S$. Пусть $\Sigma=R(T, G)$ - система корней группы $G$ относительно $T$ и $\Pi=\left\{\alpha_{1}, \ldots, \alpha_{n}\right\}$ - произвольный ее базис. Если $\left\{X_{\alpha, \alpha \in \Sigma}, H_{\alpha_{1}}, \ldots, H_{\alpha_{n}}\right\}$ - базис Шевалле алгебры Ли $\mathfrak{g}$ группы $G$, то $G$ порождается над $\bar{K}$ соответствующими корневыми подгруппами $u_{\alpha}=$ $\left\langle x_{\alpha}(t) \mid t \in \bar{K}\right\rangle$, а тор $T$ - элементами вида $h_{\alpha}(t)=w_{\alpha}(t) w_{\alpha}(1)^{-1}$, где $w_{\alpha}(t)=x_{\alpha}(t) x_{-\alpha}\left(-t^{-1}\right) x_{\alpha}(t)$ [22]. Так как $G$ - односвязная группа, то $T$ прямо порождается одномерньми подгруппами $T_{\alpha_{i}}=\left\langle h_{\alpha_{i}}(t) \mid t \in \bar{K}^{*}\right\rangle$, т.е. произвольный элемент $t \in T(\bar{K})$ однозначно записывается в виде $t=\prod_{i=1}^{n} h_{\alpha_{i}}\left(t_{i}\right)$, $t_{i} \in \bar{K}^{*}[22]$.

В приведенных выше обозначениях тор $S$ является связной компонентой решения следующей системы уравнений:

$$
\begin{cases}\alpha_{i}(t)=1 & \text { для всех необведенных вершин } \alpha_{i} K \text {-индекса Титса, } \\ \beta(t)=\sigma^{*}(\beta)(t) & \text { для всех } \beta \in \Pi \text { и всех } \sigma \in \Gamma=\operatorname{Gal}(\bar{K} / K),\end{cases}
$$


где $t \in T$ и через $\sigma^{*}$ обозначено соответствующее действие группы Галуа $\Gamma$ на системе простых корней П [21].

Если $G$ - группа внутреннего типа, то $\sigma^{*}(\beta)=\beta$ для всех $\beta \in \Pi$ [21]. Кроме того, поскольку для любых двух корней $\alpha, \beta \in \Sigma$ справедливо соотношение

$$
h_{\alpha}(t) x_{\beta}(u) h_{\alpha}(t)^{-1}=x_{\beta}\left(t^{\langle\beta, \alpha\rangle} u\right),
$$

где $\langle\beta, \alpha\rangle=2(\beta, \alpha) /(\alpha, \alpha)[21]$, то первое уравнение приведенной вьше системы сводится к

$$
\prod_{j=1}^{n} t_{j}^{2\left(\alpha_{i}, \alpha_{j}\right) /\left(\alpha_{j}, \alpha_{j}\right)}=1,
$$

где $t=\prod_{j=1}^{n} h_{\alpha_{j}}\left(t_{j}\right)$.

Перейдем к описанию группы $A$.

3.3. Тип $G_{2}, F_{4}, E_{8}$. Так как $\mu=1$, то $A=H^{1}(K, \mu)=1$.

3.4.1. Тип ${ }^{1} E_{6}: \mu=\mu_{3}$, и поэтому $H^{1}(K, \mu)$ можно отождествить с $K^{*} /\left(K^{*}\right)^{3}$. Очевидно, что для всех $v \in V_{\infty}$ имеется равенство

$$
H^{1}\left(K_{v}, \mu_{3}\right)=K_{v}^{*} /\left(K_{v}^{*}\right)^{3}=1
$$

откуда следует, что

$$
A=H^{1}(K, \mu) .
$$

3.4.2. Тип ${ }^{2} E_{6}: \mu=\mathbf{R}_{L / K}^{(1)}\left(\mu_{3}\right),[L: K]=2$. Группа $H^{1}(K, \mu)$ входит в следуюшую точную последовательность [12]:

$$
1 \rightarrow \mu_{3}(K) / \mathrm{N}_{L / K}\left(\mu_{3}(L)\right) \rightarrow H^{1}(K, \mu) \rightarrow \operatorname{Ker}\left(L^{*} /\left(L^{*}\right)^{3} \stackrel{\mathrm{N}_{L / K}}{\longrightarrow} K^{*} /\left(K^{*}\right)^{3}\right) \rightarrow 1 .
$$

Так как $[L: K]=2$, то $\mu_{3}(K) / \mathrm{N}_{L / K}\left(\mu_{3}(L)\right)=1$, откуда

$$
H^{1}(K, \mu) \simeq \operatorname{Ker}\left(L^{*} /\left(L^{*}\right)^{3} \stackrel{\mathrm{N}_{L / K}}{\longrightarrow} K^{*} /\left(K^{*}\right)^{3}\right)
$$

Рассмотрим локальную группу $H^{1}\left(K_{v}, \mu\right)$, где $v \in V_{\infty}$ и $K_{v} \simeq \mathbb{R}$. Имеются две возможности: над полем $K_{v}$ группа $G$ имеет либо внутренний, либо внешний тип. В первом случае согласно 3.4.1 справедливо равенство $H^{1}\left(K_{v}, \mu\right)=1$. Во втором случае, очевидно, что

$$
\begin{aligned}
\operatorname{Ker}\left(\left(L \otimes_{K} K_{v}\right)^{*} /\left(L \otimes_{K} K_{v}\right)^{* 3} \stackrel{\mathrm{N}_{L \otimes_{K} K_{v} / K}}{\longrightarrow} K_{v}^{*} /\left(K_{v}^{*}\right)^{3}\right) & \\
= & \operatorname{Ker}\left(\mathbb{C}^{*} /\left(\mathbb{C}^{*}\right)^{3} \stackrel{\mathrm{N}_{\mathbb{C} / \mathbb{R}}}{\longrightarrow} \mathbb{R}^{*} /\left(\mathbb{R}^{*}\right)^{3}\right)=1 .
\end{aligned}
$$

Итак, для всех $v \in V_{\infty}$ локальные групшы $H^{1}\left(K_{v}, \mu\right)$ тривиальны, и следовательно,

$$
A=H^{1}(K, \mu) .
$$


3.5. Тип $E_{7}: \mu=\mu_{2}, H^{1}(K, \mu)$ отождествляется с $K^{*} /\left(K^{*}\right)^{2}$. Пусть $v \in V_{\infty}$ и $K_{v} \simeq \mathbb{R}$. Над полем $K_{v}$ для $K_{v}$-индекса Титса группы $G$ имеются четыре возможности [21].

a)

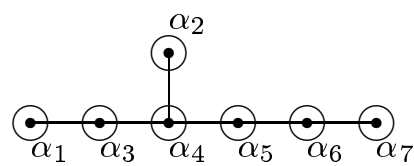

б)

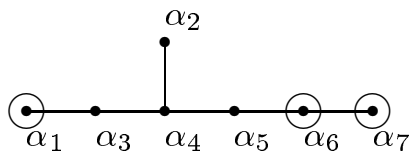

в)

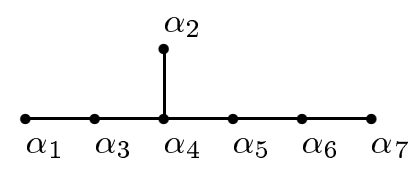

г)

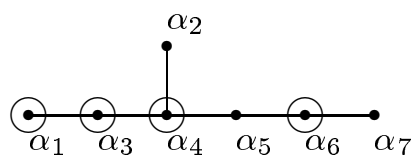

В случае а) группа $G$ разложима над $K_{v}$, откуда центр $\mu$ лежит в максимальном $K_{v}$-разложимом торе, когомологии которого тривиальны, и следовательно,

$$
\operatorname{Ker}\left(H^{1}\left(K_{v}, \mu\right) \rightarrow H^{1}\left(K_{v}, G\right)\right)=H^{1}\left(K_{v}, \mu\right) .
$$

В случае б), применяя алгоритм, приведенный в 3.2, легко убедиться, что

$$
S=\left\langle h_{\alpha_{1}}\left(t_{1}^{2} / t_{2}^{2}\right) h_{\alpha_{2}}\left(t_{2}\right) h_{\alpha_{3}}\left(t_{1}\right) h_{\alpha_{4}}\left(t_{2}^{2}\right) h_{\alpha_{5}}\left(t_{2}^{3} / t_{1}\right) h_{\alpha_{6}}\left(t_{2}^{4} / t_{1}^{2}\right) h_{\alpha_{7}}\left(t_{3}\right) \mid t_{i} \in \bar{K}_{v}^{*}\right\rangle .
$$

Так как $\mu$ порождается элементом $h_{\alpha_{2}}(-1) h_{\alpha_{5}}(-1) h_{\alpha_{7}}(-1)$, то $\mu \leqslant S$. Отсюда следует, что и здесь справедливо равенство (1).

В случае в) группа $G$ является $K_{v}$-анизотропной, и следовательно, морфизм $G(\mathbb{R}) \rightarrow \bar{G}(\mathbb{R})$ сюръективен [12], откуда

$$
\operatorname{Ker}\left(H^{1}\left(K_{v}, \mu\right) \rightarrow H^{1}\left(K_{v}, G\right)\right)=1
$$

Наконец, рассмотрим случайг). Покажем, что так же,как и в случаев), морфизм $G(\mathbb{R}) \rightarrow \bar{G}(\mathbb{R})$ сюръективен. Пусть $S$ - максимальный $K_{v}$-разложимый тор в $G$, $\bar{S}$ - его образ в присоединенной группе $\bar{G}$. Из разложения Брюа-Титса следует, что достаточно проверить сюръективность морфизма $Z_{G}(S)(\mathbb{R}) \rightarrow Z_{\bar{G}}(\bar{S})(\mathbb{R})$. С этой целью рассмотрим следуюшую коммутативную диаграмму с точными столбцами:

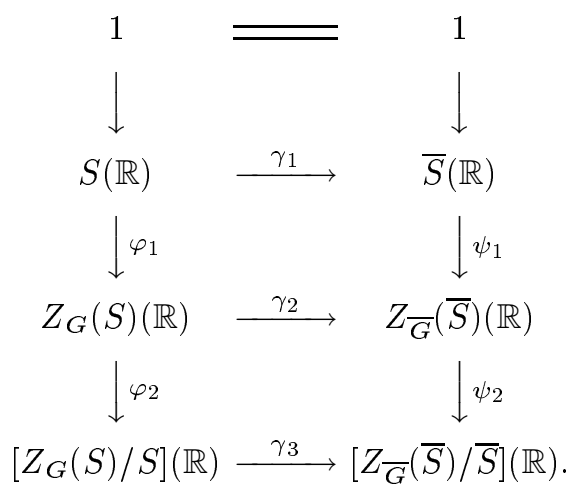


Из когомологической тривиальности торов $S, \bar{S}$ следует, что морфизмы $\gamma_{2}, \psi_{2}$ сюръективны. Поэтому для доказательства сюръективности $\varphi_{2}$ достаточно установить сюръективность $\gamma_{3}, \gamma_{1}$.

Применяя алгоритм из 3.2, получим, что

$$
S=\left\langle h_{\alpha_{1}}\left(t_{1}\right) h_{\alpha_{4}}\left(t_{1}^{2}\right) h_{\alpha_{5}}\left(t_{1} t_{2}\right) h_{\alpha_{6}}\left(t_{2}^{2}\right) h_{\alpha_{7}}\left(t_{2}\right) h_{\alpha_{1}}\left(t_{3}\right) h_{\alpha_{3}}\left(t_{4}\right) \mid t_{i} \in \bar{K}^{*}\right\rangle .
$$

Отсюда следует, что $\mu \nless S$, и в частности, $\gamma_{1}-$ изоморфизм. Наконец, сюръективность $\gamma_{3}$ - это общий факт [12], так как $C_{G}(S) / S, C_{\bar{G}}(\bar{S}) / \bar{S}$ - полупростые компактные групшы.

Таким образом, суммируя все вместе, получаем следуюшее описание группы $A$ :

$$
\begin{aligned}
A \simeq\left\{a K^{* 2} \in K^{*} /\left(K^{*}\right)^{2} \mid\right. & a \text { положителен в } K_{v} \text { для всех } v \in V_{\infty} \text { таких, } \\
\text { что } K_{v} & \left.\left.\simeq \mathbb{R} \text { и } K_{v} \text {-индекс Титса имеет вид в }\right), \text { г) }\right\} .
\end{aligned}
$$

ЗАмечАниЕ. Анализ приведенного вьше доказательства показывает, что имеет место следуюшее утверждение. Пусть $G$ - односвязная алгебраическая группа, определенная над полем $\mathbb{R}, \mu$ - ее центр, $\bar{G}$ - соответствующая присоединенная группа, $S$ - максимальный $\mathbb{R}$-разложимый тор группы $G, \bar{S}$ - его образ в $\bar{G}$. Тогда из сюръективности морфизма $\gamma_{1}: S(\mathbb{R}) \rightarrow \bar{S}(\mathbb{R})$ следует сюръективность морфизма $Z_{G}(S)(\mathbb{R}) \rightarrow Z_{\bar{G}}(\bar{S})(\mathbb{R})$, а значит, и сюръективность $G(\mathbb{R}) \rightarrow \bar{G}(\mathbb{R})$. Последнее означает, что в этом случае справедливо равенство (2). В частности, если $\mu \cap S=1$, то $\gamma_{1}$ - всегда сюръективный морфизм, и следовательно, справедливо (2).

3.6.1. Тип ${ }^{1} A_{2 n}: \mu=\mu_{2 n+1}, H^{1}(K, \mu)$ можно отождествить с $K^{*} /\left(K^{*}\right)^{2 n+1}$. Поскольку локальные групшы $H^{1}\left(K_{v}, \mu\right)$ тривиальны для всех $v \in V_{\infty}$, то

$$
A=H^{1}(K, \mu) \text {. }
$$

3.6.2. Тип ${ }^{2} A_{2 n}: \mu \simeq \mathbf{R}_{L / K}^{(1)}\left(\mu_{2 n+1}\right)$ и группа когомологий центра входит в следующую точную последовательность [12]

$$
\begin{aligned}
1 \rightarrow \mu_{2 n+1}(K) / \mathrm{N}_{L / K}\left(\mu_{2 n+1}(L)\right) & \rightarrow H^{1}(K, \mu) \\
\rightarrow & \operatorname{Ker}\left(L^{*} /\left(L^{*}\right)^{2 n+1} \stackrel{\mathrm{N}_{L / K}}{\longrightarrow} K^{*} /\left(K^{*}\right)^{2 n+1}\right) \rightarrow 1 .
\end{aligned}
$$

Так как $[L: K]=2$, то левый член этой последовательности тривиален, и поэтому можно считать, что

$$
H^{1}(K, \mu) \simeq \operatorname{Ker}\left(L^{*} /\left(L^{*}\right)^{2 n+1} \stackrel{\mathrm{N}_{L / K}}{\longrightarrow} K^{*} /\left(K^{*}\right)^{2 n+1}\right) .
$$

Пусть $v \in V_{\infty}$ и $K_{v} \simeq \mathbb{R}$. Если $G$ над полем $K_{v}$ имеет внутренний тип, то согласно 3.6.1 группа $H^{1}\left(K_{v}, \mu\right)$ тривиальна. Если же над $K_{v}$ группа $G$ остается группой внешнего типа, то

$$
\left(L \otimes_{K} K_{v}\right)^{*} /\left(L \otimes_{K} K_{v}\right)^{* 2 n+1} \simeq \mathbb{C}^{*} /\left(\mathbb{C}^{*}\right)^{2 n+1}=1,
$$

и следовательно, $H^{1}\left(K_{v}, \mu\right)$ также тривиальна. В результате имеем:

$$
A=H^{1}(K, \mu) .
$$


3.7.1. Тип ${ }^{1} A_{2 n-1}: \mu=\mu_{2 n}, H^{1}(K, \mu) \simeq K^{*} /\left(K^{*}\right)^{2 n}$. Пусть $v \in V_{\infty}$ и $K_{v} \simeq \mathbb{R}$. Случай, когда $G$ разложима над $K_{v}$, разбирается аналогично случаю групп типа $E_{7}$, и поэтому здесь и далее для групп типов $B_{n}, C_{n}, D_{n}$ рассматриваться не будет.

Пусть $G$ неразложима над $K_{v}$. Тогда она изоморфна над $K_{v}$ группе вида $\mathrm{SL}(1, A)$, где $A=M_{n}(D)$ и $D$ - тело вешественных кватернионов [21]. Поэтому $H^{1}\left(K_{v}, G\right)$ можно отождествить с

$$
K_{v}^{*} / \operatorname{Nrd}\left(M_{n}(D)\right) \simeq K_{v}^{*} / \operatorname{Nrd}(D) \simeq \mathbb{R}^{*} /\left(\mathbb{R}^{*}\right)^{2} .
$$

При этом отождествлении морфизм $H^{1}\left(K_{v}, \mu\right) \rightarrow H^{1}\left(K_{v}, G\right)$ соответствует тождественному морфизму $\mathbb{R}^{*} /\left(\mathbb{R}^{*}\right)^{2} \rightarrow \mathbb{R}^{*} /\left(\mathbb{R}^{*}\right)^{2 n}$, и значит, выполняется равенство (2).

Итак, доказано, что

$$
\begin{array}{r}
A \simeq\left\{a K * \in K^{*} /\left(K^{*}\right)^{2 n} \mid a \text { положителен в } K_{v} \text { для всех } v \in V_{\infty}\right. \text { таких, } \\
\text { что } \left.G \text { неразложима над } K_{v}\right\} .
\end{array}
$$

3.7.2. Тип ${ }^{2} A_{2 n-1}: \mu \simeq \mathbf{R}_{L / K}^{(1)}\left(\mu_{2 n}\right)$ и $H^{1}(K, \mu)$ входит в следуюшую точную последовательность:

$$
\begin{aligned}
1 \rightarrow \mu_{2 n}(K) / \mathrm{N}_{L / K}\left(\mu_{2 n}(L)\right) & \stackrel{\varphi_{1}}{\longrightarrow} H^{1}(K, \mu) \\
& \stackrel{\varphi_{2}}{\longrightarrow} \operatorname{Ker}\left(L^{*} /\left(L^{*}\right)^{2 n} \stackrel{\mathrm{N}_{L / K}}{\longrightarrow} K^{*} /\left(K^{*}\right)^{2 n}\right) \rightarrow 1 .
\end{aligned}
$$

Пусть $v \in V_{\infty}$ и $K_{v} \simeq \mathbb{R}$. Для $K_{v}$-индекса Титса имеются четыре возможности [21].

a)

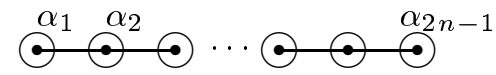

б)

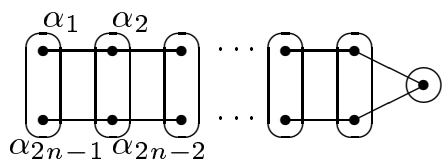

в)

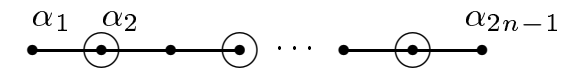

г)

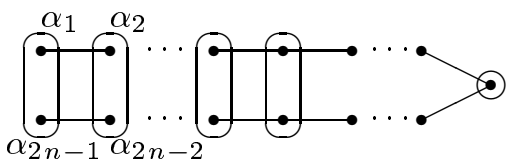

Пусть $S$ - максимальный $K_{v}$-разложимый тор в $G$. Случаи а), б) соответствуют разложимому и квазиразложимому случаю, для которых централизатор $Z_{G}(S)$ является максимальным тором с тривиальными когомологиями. Тем самым для а), б) справедливо равенство (1).

Рассмотрим случай в). Напомним [12], что последовательность (4) индуцировалась точной последовательностью

$$
1 \rightarrow \mathbf{R}_{L / K}^{(1)}\left(\mu_{2 n}\right) \rightarrow \mathbf{R}_{L / K}\left(\mu_{2 n}\right) \stackrel{\mathrm{N}_{L / K}}{\longrightarrow} \mu_{2 n} \rightarrow 1 .
$$


Так как группа $G$ над полем $K_{v}$ имеет внутренний тип, то

$$
\left(\mathbf{R}_{L \otimes K_{v} / K_{v}}\left(\mu_{2 n}\right)\right)\left(K_{v}\right)=\mu_{2 n}\left(K_{v}\right) \times \mu_{2 n}\left(K_{v}\right) .
$$

Отсюда следует, что морфизм $\mathrm{N}_{L / K}$ в последовательности (5) сюръективен на $K_{v}$-точках. Это означает, что левый член в последовательности (4) при морфизме ограничения $\operatorname{Res}_{K_{v}}$ становится тривиальньм, откуда

$$
H^{1}\left(K_{v}, \mu\right) \simeq \operatorname{Ker}\left(\left(L \otimes_{K} K_{v}\right)^{*} /\left(L \otimes_{K} K_{v}\right)^{* 2 n} \stackrel{\mathrm{N}_{L \otimes K_{v} / K_{v}}}{\longrightarrow} K_{v}^{*} /\left(K_{v}^{*}\right)^{2 n}\right) .
$$

В свою очередь, так как $L \otimes_{K} K_{v} \simeq K_{v} \times K_{v}$, то правую часть (6) можно отождествить с $K_{v}^{*} /\left(K_{v}^{*}\right)^{2 n}=\mathbb{R}^{*} /\left(\mathbb{R}^{*}\right)^{2}$. Тогда, применяя 3.7.1, получаем, что ограничение коцикла $\left(a_{\sigma}\right) \in Z^{1}(K, \mu)$ тривиально в $H^{1}\left(K_{v}, G\right)$ тогда и только тогда, когда $\operatorname{Res}_{K_{v}}\left[\varphi_{2}\left(\left(a_{\sigma}\right)\right)\right]$ - положительное число в $K_{v}$ (при указанном отождествлении). Отметим, что последнее эквивалентно тому, что $\operatorname{Res}_{K_{v}}\left(a_{\sigma}\right)$ тривиален в $H^{1}\left(K_{v}, \mu\right)$.

Перейдем к случаю г). В этом случае группа $G$ над полем $K_{v}$ имеет внешний тип, откуда $L \otimes_{K} K_{v} \simeq \mathbb{C}$, и следовательно,

$$
\operatorname{Ker}\left(\left(L \otimes_{K} K_{v}\right)^{*} /\left(L \otimes_{K} K_{v}\right)^{* 2 n} \stackrel{\mathrm{N}_{L \otimes K_{v} / K_{v}}}{\longrightarrow} K_{v}^{*} /\left(K_{v}^{*}\right)^{2 n}\right)=1 .
$$

Поэтому

$$
H^{1}\left(K_{v}, \mu\right) \simeq \mu_{2 n}\left(K_{v}\right) / \mathrm{N}_{L \otimes_{K} K_{v} / K_{v}}\left(\mu_{2 n}\left(L \otimes_{K} K_{v}\right)\right)=\langle \pm 1\rangle
$$

- группа порядка 2. Пользуясь алгоритмом, указанным в 3.2 , легко подсчитать, что

$S=\left\langle h_{\alpha_{1}}\left(t_{1}\right) h_{\alpha_{2 n-1}}\left(t_{1}\right) \cdots h_{\alpha_{r}}\left(t_{r}\right) h_{\alpha_{2 n-r}}\left(t_{r}\right) h_{\alpha_{r+1}}\left(t_{r}\right) \cdots h_{\alpha_{2 n-r-1}}\left(t_{r}\right) \mid t_{i} \in \bar{K}^{*}\right\rangle$.

Отсюда следует, что $S \cap \mu=1$, ибо $\mu$ порождается элементом

$$
h_{\alpha_{1}}(\zeta) h_{\alpha_{2}}\left(\zeta^{2}\right) \cdots h_{\alpha_{2 n-1}}\left(\zeta^{2 n-1}\right)
$$

где $\zeta$ - первообразный корень из 1 степени $2 n$. Тогда из замечания 3.5 вытекает, что морфизм $G(\mathbb{R}) \rightarrow \bar{G}(\mathbb{R})$ - сюръективен, и значит, выполняется равенство (2).

Итак, имеем:

$A=\left\{x \in H^{1}(K, \mu) \mid \operatorname{Res}_{K_{v}}(x)=1 \in H^{1}\left(K_{v}, \mu\right)\right.$ для всех $v \in V_{\infty}$ таких, что $K_{v} \simeq \mathbb{R}$ и $G$ не является ни разложимой, ни квазиразложимой над $\left.K_{v}\right\}$. 
3.8. Тип $B_{n}: \mu=\mu_{2}, H^{1}(K, \mu) \simeq K^{*} /\left(K^{*}\right)^{2}$. Из описания групп этой серии следует [21], что $G \simeq \operatorname{Spin}(f)$, где $f-(2 n+1)$-мерная невырожденная квадратичная форма, определенная над полем $K$.

Пусть $v \in V_{\infty}$ и $K_{v} \simeq \mathbb{R}$. Хорошо известно, что образ морфизма

$$
\varphi: G(\mathbb{R})=\operatorname{Spin}(f)(\mathbb{R}) \rightarrow \bar{G}(\mathbb{R})=\mathrm{SO}(f)(\mathbb{R})
$$

состоит из ортогональных преобразований, спинорная норма которых является квадратом. Отсюда следует, что $\varphi$ - сюръективный морфизм тогда и только тогда, когда $f$ - анизотропна над $K_{v}$, или же, что эквивалентно, $G$ - анизотропна над $K_{v}$. Поэтому имеем:

$$
\begin{array}{r}
A=\left\{a\left(K^{*}\right)^{2} \in K^{*} /\left(K^{*}\right)^{2} \mid a \text { положителен в } K_{v} \text { для всех } v \in V_{\infty}\right. \text { таких, } \\
\text { что } \left.G \text { анизотропна над } K_{v}\right\} .
\end{array}
$$

3.9. Тип $C_{n}: \mu=\mu_{2}, H^{1}(K, \mu) \simeq K^{*} /\left(K^{*}\right)^{2}$. Пусть $v \in V_{\infty}, K_{v} \simeq \mathbb{R}$ и $G$ не является $K_{v}$-разложимой. Тогда над полем $K_{v}$ группа $G$ изоморфна группе $\mathrm{SU}_{n}(D, h)$, где $D$ - тело вешественных кватернионов, $h$ - невырожденная эрмитова форма относительно стандартной инволюции $\tau$ [21].

Далее следует различать два случая: индекс Витта $r$ формы $h$ а) либо совпадает с $n / 2$ (в частности, $n$ должно быть четньм), б) либо строго меншше $n / 2$. В первом случае $K_{v}$-индекс Титса имеет вид

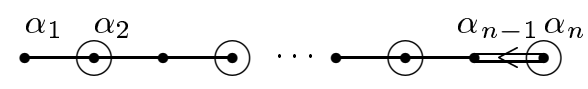

а во втором

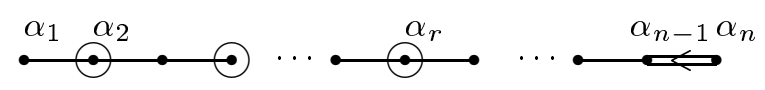

Легко проверить, что в первом случае центр $\mu$ лежит в максимальном $K_{v}$-разложимом торе $S$, а во втором - не лежит. Поэтому имеем:

$$
\begin{array}{r}
A=\left\{a\left(K^{*}\right)^{2} \in K^{*} /\left(K^{*}\right)^{2} \mid a \text { положителен в } K_{v} \text { для всех } v \in V_{\infty}\right. \text { таких, } \\
\text { что } \left.K_{v} \text {-индекс Титса имеет вид (7) }\right\} .
\end{array}
$$

3.10.1. Тип ${ }^{1} D_{2 n+1}: \mu=\mu_{4}, H^{1}(K, \mu)=K^{*} /\left(K^{*}\right)^{4}$. Пусть $v \in V_{\infty}$ и $K_{v} \simeq \mathbb{R}$. Если $G$ над $K_{v}$ анизотропна, то $G(\mathbb{R}) \rightarrow \bar{G}(\mathbb{R})$ - сюръективный морфизм, и значит, справедливо равенство (2).

Пусть $G$ - изотропна над $K_{v}$. Тогда для $K_{v}$-индекса Титса имеются две возможности:

a)

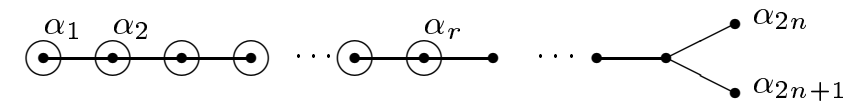

б)

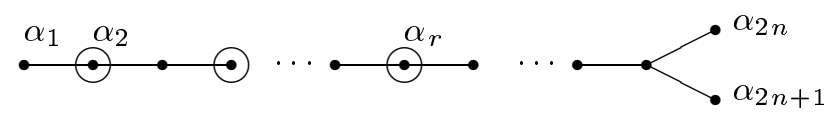


В случае а) группа $G$ над полем $K_{v}$ изоморфна спинорной группе $\operatorname{Spin}(f)$, связанной с $K_{v}$-изотропной квадратичной формой $f$. Тогда морфизм

$$
\operatorname{Spin}(f)(\mathbb{R}) \rightarrow \operatorname{SO}(f)(\mathbb{R})
$$

несюръективен, что влечет также несюръективность морфизма $G(\mathbb{R}) \rightarrow \bar{G}(\mathbb{R})$, и следовательно, в случае а) справедливо равенство (1), так как $\left|H^{1}\left(K_{v}, \mu\right)\right|=2$.

В случае б) легко проверить, что $S \cap \mu=1$, и поэтому справедливо равенство (2).

В результате имеем следующее описание группы $A$ :

$$
\begin{gathered}
A=\left\{a\left(K^{*}\right)^{4} \in K^{*} /\left(K^{*}\right)^{4} \mid a \text { положителен в } K_{v} \text { для всех } v \in V_{\infty}\right. \text { таких, что } \\
\text { либо } \left.G \text { анизотропна над } K_{v}, \text { либо ее } K_{v} \text {-индекс Титса имеет вид }(9)\right\} .
\end{gathered}
$$

3.10.2. Тип ${ }^{2} D_{2 n+1}: \mu=\mathbf{R}_{L / K}^{(1)}\left(\mu_{4}\right)$. Над вещественными пополнениями для $K_{v}$-изотропных групп $K_{v}$-индекс Титса имеет вид либо (8), либо (9), либо

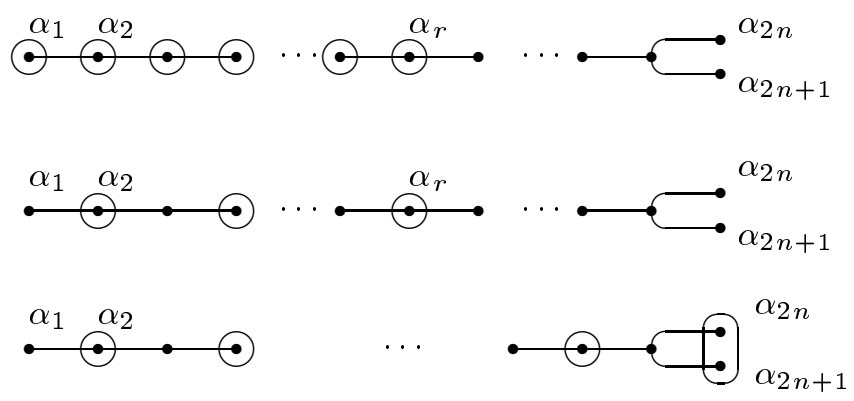

Группа $H^{1}(K, \mu)$ входит в следующую точную последовательность:

$1 \rightarrow \mu_{4}(K) / \mathrm{N}_{(L / K)}\left(\mu_{4}(L)\right) \rightarrow H^{1}(K, \mu) \rightarrow \operatorname{Ker}\left(L^{*} /\left(L^{*}\right)^{4} \stackrel{\mathrm{N}_{L / K}}{\longrightarrow} K^{*} /\left(K^{*}\right)^{4}\right) \rightarrow 1$.

Отсюда следует, что $\left|H^{1}\left(K_{v}, \mu\right)\right|=2$ в случаях $(10),(11),(12)$, и кроме того, так же, как и в 3.10 .1 , показывается, что в случае $(10)$ морфизм $G(\mathbb{R}) \rightarrow \bar{G}(\mathbb{R})$ несюръективен, а в случаях (11), (12) - сюръективен. Это означает, что для случая (10) вьполняется равенство (1), а для (11), (12) - равенство (2).

В результате имеем:

$A=\left\{x \in H^{1}(K, \mu) \mid \operatorname{Res}_{K_{v}}(x)\right.$ - положительное число для всех $v \in V_{\infty}$ таких, что либо $G$ анизотропна над $K_{v}$, либо ее $K_{v}$-индекс Титса имеет вид $\left.(9),(11),(12)\right\}$.

Отметим, что слова "Res $K_{v}(x)$ - положительное число" означают, что $H^{1}\left(K_{v}, \mu\right)$ отождествляется с $\mu_{4}\left(K_{v}\right) / \mathrm{N}_{\left(L \otimes_{K} K_{v} / K_{v}\right)}\left(\mu_{4}\left(L \otimes_{K} K_{v}\right)\right)=\langle \pm 1\rangle$, если $G$ над $K_{v}$ имеет внешний тип, и с

$$
\begin{aligned}
\operatorname{Ker}\left(\left(L \otimes_{K} K_{v}\right)^{*} /\left(\left(L \otimes_{K} K_{v}\right)^{*}\right)^{4}\right. & \left.\rightarrow K_{v}^{*} /\left(K_{v}^{*}\right)^{4}\right) \\
& =\operatorname{Ker}\left(\left(K_{v}^{*} \times K_{v}^{*}\right) /\left(K_{v}^{* 4} \times K_{v}^{* 4}\right) \rightarrow K_{v}^{*} /\left(K_{v}^{*}\right)^{4}\right) \simeq \mathbb{R}^{*} / \mathbb{R}^{* 4}=\mathbb{R}^{*} / \mathbb{R}^{* 2},
\end{aligned}
$$

если $G$ имеет внутренний тип. 
3.11.1. Тип ${ }^{1} D_{2 n}$. Занумеруем вершины графа Дьнкина следуюшим образом:

Тогда $\mu=\mu_{1} \times \mu_{2}$, где

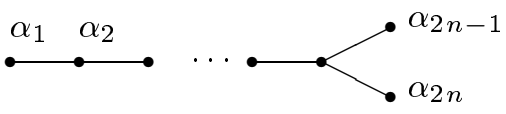

$$
\begin{aligned}
& \mu_{1}=\left\langle h_{\alpha_{1}}(-1) h_{\alpha_{3}}(-1) \cdots h_{\alpha_{2 n-1}}(-1)\right\rangle, \\
& \mu_{2}=\left\langle h_{\alpha_{1}}(-1) h_{\alpha_{3}}(-1) \cdots h_{\alpha_{2 n-3}}(-1) h_{\alpha_{2 n}}(-1)\right\rangle .
\end{aligned}
$$

Отождествим $H^{1}\left(K_{v}, \mu_{i}\right)$ с $K^{*} /\left(K^{*}\right)^{2}$, а $H^{1}(K, \mu)-$ с $H^{1}\left(K, \mu_{1}\right) \times H^{1}\left(K, \mu_{2}\right)$.

Пусть $v \in V_{\infty}, K_{v} \simeq \mathbb{R}$ и группа $G$ изотропна над $K_{v}$, но не разложима. Тогда имеются следуюшие две возможности.

1) $G \stackrel{K_{v}}{\simeq} \operatorname{Spin}(f)$, где $f-K_{v}$-изотропная, но не разложимая, квадратичная форма размерности $4 n$. В этом случае $K_{v}$-индекс Титса имеет вид [21]:

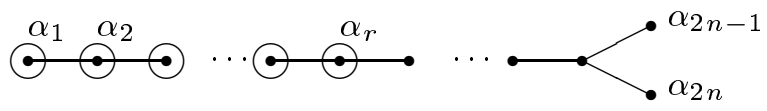

Морфизм $G(\mathbb{R}) \rightarrow \bar{G}(\mathbb{R})$ разлагается в композицию морфизмов

$$
\varphi_{1}: \operatorname{Spin}(f)(\mathbb{R}) \rightarrow \mathrm{SO}(f)(\mathbb{R}), \quad \varphi_{2}: \mathrm{SO}(f)(\mathbb{R}) \rightarrow \operatorname{PSO}(f)(\mathbb{R}),
$$

причем очевидно, что первый из них не сюръективен, а второй - сюръективен (см. замечание 3.5$)$; в частности, $\left[\bar{G}(\mathbb{R}):\left(\varphi_{1} \circ \varphi_{2}\right)(G(\mathbb{R}))\right]=2$, откуда

$$
\left|\operatorname{Ker}\left(H^{1}\left(K_{v}, \mu\right) \rightarrow H^{1}\left(K_{v}, G\right)\right)\right|=2 .
$$

В корневых терминах морфизм $\varphi_{1}$ соответствует морфизму

$$
G \rightarrow G /\left\langle h_{\alpha_{2 n-1}}(-1) h_{\alpha_{2 n}}(-1)\right\rangle .
$$

Поэтому

$$
\begin{aligned}
& \operatorname{Ker}\left(H^{1}\left(K_{v}, \mu\right) \rightarrow H^{1}\left(K_{v}, G\right)\right) \\
& \quad=\left\{\left(a K_{v}^{* 2}, b K_{v}^{* 2}\right) \in K_{v}^{*} /\left(K_{v}^{*}\right)^{2} \times K_{v}^{*} /\left(K_{v}^{*}\right)^{2} \mid a, b \text { имеют один знак }\right\} .
\end{aligned}
$$

2) $G \simeq \operatorname{Spin}(D, h)$, где $D$ - тело вешественных кватернионов, а $h$ - изотропная косоэрмитова форма относительно стандартной инволюции $\tau$ тела $D$. Если индекс Витта $r$ формы $h$ принимает максимально возможное значение, равное $n$, то $K_{v}$-индекс Титса имеет вид

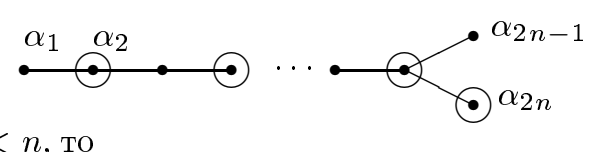

если же $r<n$, то

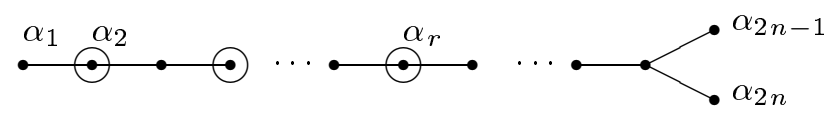


В случае (14) так же, как и в 1$)$, показывается, что $[\bar{G}(\mathbb{R}): \varphi(G(\mathbb{R})]=2$, где $\varphi: G \rightarrow \bar{G}$ - канонический морфизм, откуда

$$
\left|\operatorname{Ker}\left(H^{1}\left(K_{v}, \mu\right) \rightarrow H^{1}\left(K_{v}, G\right)\right)\right|=2 .
$$

Чтобы получить точное описание этого ядра, следует рассмотреть два подслучая: $n$ четно и $n$ нечетно.

Если $n$ четно, то, используя алгоритм 3.2 , проверяется, что $\mu_{1}$ лежит в максимальном $K_{v}$-разложимом торе $S$. Это означает, что морфизм $G(\mathbb{R}) \rightarrow\left(G / \mu_{1}\right)(\mathbb{R})$ несюръективен, и значит,

$$
\operatorname{Ker}\left(H^{1}\left(K_{v}, \mu\right) \rightarrow H^{1}\left(K_{v}, G\right)\right)=H^{1}\left(K_{v}, \mu_{1}\right) .
$$

Если же $n$ нечетно, то $\mu_{2} \leqslant S$, откуда

$$
\operatorname{Ker}\left(H^{1}\left(K_{v}, \mu\right) \rightarrow H^{1}\left(K_{v}, G\right)\right)=H^{1}\left(K_{v}, \mu_{2}\right) .
$$

В случае (15) легко проверить, что $S \cap \mu=1$, и следовательно, выполняется равенство (2).

Подытоживая, имеем: $A=\left\{\left(\left(a\left(K^{*}\right)^{2}, b\left(K^{*}\right)^{2}\right) \in K^{*} /\left(K^{*}\right)^{2} \times K^{*} /\left(K^{*}\right)^{2}\right) \mid\right.$ для всех $v \in V_{\infty}$ таких, что $K_{v} \simeq \mathbb{R}$, выполняется: $a, b>0$, если $G-K_{v}$-анизотропна; $a \cdot b>0$, если $K_{v}$-индекс Титса имеет вид (13); $a, b>0$, если $K_{v}$-индекс Титса имеет вид $(15) ; b>0$, если $K_{v}$-индекс Титса имеет вид (14) и $n$ четно; $a>0$, если $K_{v}$-индекс Титса имеет вид (14) и $n$ нечетно $\}$.

3.11.2. Тип ${ }^{2} D_{2 n}: \mu=\mathbf{R}_{L / K}\left(\mu_{2}\right), H^{1}(K, \mu) \simeq L^{*} /\left(L^{*}\right)^{2}$. Если $v \in V_{\infty}$, $K_{v} \simeq \mathbb{R}$ и $G$ над $K_{v}$ имеет внешний тип, то $H^{1}\left(K_{v}, \mu\right)=1$, ибо $L \otimes_{K} K_{v} \simeq \mathbb{C}$. Поэтому с учетом 3.11.1 имеем $A=\left\{\left(a\left(L^{*}\right)^{2} \in L^{*} /\left(L^{*}\right)^{2}\right) \mid\right.$ для всех $v \in V_{\infty}$ таких, что $K_{v} \simeq \mathbb{R}$ и $G$ имеет над $K_{v}$ внутренний тип, вьполняется: $a, \sigma(a)>0$, если $G-K_{v}$-анизотропна; $\mathrm{N}_{L / K}(a)>0$, если $K_{v}$-индекс Титса имеет вид (13); $a, \sigma(a)>0$, если $K_{v}$-индекс Титса имеет вид $(15) ; \sigma(a)>0$, если $K_{v}$-индекс Титса имеет вид (14) и $n$ четно; $a>0$, если $K_{v}$-индекс Титса имеет вид (14) и $n$ нечетно $\}$, где через $\sigma$ обозначается нетривиальный автоморфизм $L$ над $K$.

3.12. Тип ${ }^{3,6} D_{4}: \mu=\mathbf{R}_{P / K}^{(1)}\left(\mu_{2}\right)$, где $P=L$, если $L$ - расширение Галуа над $K$ степени 3, и $P$ - произвольное подполе в $L$ степени 3 над $K$, если $[L: K]=6$.

$H^{1}(K, \mu)$ входит в следуюшую точную последовательность:

$$
1 \rightarrow \mu_{2}(K) / \mathrm{N}_{P / K}\left(\mu_{2}(P)\right) \rightarrow H^{1}(K, \mu) \rightarrow \operatorname{Ker}\left(P^{*} /\left(P^{*}\right)^{2} \stackrel{\mathrm{N}_{P / K}}{\longrightarrow} K^{*} /\left(K^{*}\right)^{2}\right) \rightarrow 1 .
$$

Левый член этой последовательности тривиален, и поэтому

$$
H^{1}(K, \mu) \simeq \operatorname{Ker}\left(P^{*} /\left(P^{*}\right)^{2} \rightarrow K^{*} /\left(K^{*}\right)^{2}\right) .
$$

Ясно, что над архимедовыми пополнениями группа $G$ является группой типа ${ }^{1,2} D_{4}$. Обозначим через $\sigma$ произвольньй элемент группы $\mathrm{Gal}(L / K)$ третьего порядка. Тогда из 3.11.1, 3.11.2 имеем: $A=\left\{\left(a\left(P^{*}\right)^{2} \in P^{*} /\left(P^{*}\right)^{2}\right) \mid \mathrm{N}_{P / K}(a) \in\left(K^{*}\right)^{2}\right.$ и для всех $v \in V_{\infty}$ таких, что $K_{v} \simeq \mathbb{R}$ и $G$ имеет над $K_{v}$ внутренний тип, выполняется: $a, \sigma(a)>0$, если $G-K_{v}$-анизотропна; $a \sigma(a)>0$, если $K_{v}$-индекс Титса имеет вид (13); $a, \sigma(a)>0$, если $K_{v}$-индекс Титса имеет вид $(15) ; \sigma(a)>0$, если $K_{v}$-индекс Титса имеет вид (14) $\}$. 


\section{§4. Классификация МАП групп исключительных типов}

4.1. Пусть $G$ - односвязная (абсолютно) простая алгебраическая групша, определенная над $K$. Далее мы увидим, что чем меньше центр $\mu$ группы $G$, тем проще классификация ее МАП. Поэтому разберем вначале наиболее простой случай групп исключительных типов.

Согласно теореме 2.6 представителями классов сопряженных МАП являются группы вида $\Gamma_{X}=N_{G(\bar{K})}\left(P_{X}\right)$, где $P_{X}$ - стандартная парахорическая подгруппа группы $G(K) \quad \mathscr{O}$-максимального типа $X$. Отсюда следует, что для получения классификации МАП достаточно научиться описывать всевозможные максимальные типы $X=\left\{X_{v}\right\}_{v \in V_{f}}$ и среди них уметь выделять $\mathscr{O}$-максимальные. Для этого, в свою очередь, надо знать строение локальных групп автоморфизмов $\Xi_{v}=\xi_{v}\left(H^{1}\left(K_{v}, \mu\right)\right)$ (см. 1.8) и их действия на соответствующих локальных диаграммах Дынкина $\Delta_{v}$ [23]. Напомним, что локальная диаграмма Дынкина $\Delta_{v}$ групшы $G\left(K_{v}\right)$ получается из графа Кокстера приписьванием некоторым его вершинам положительных чисел или символов “×”, "s", " $h s$ ", а некоторьм ребрам направления [23].

4.2. Если группа $G$ разложима или квазиразложима над $K_{v}$, то для описания групшы $\Xi_{v}$ проше всего воспользоваться изоморфизмом [23]

$$
\Xi_{v} \simeq \bar{S}\left(K_{v}\right) /\left(\pi\left(S\left(K_{v}\right)\right) \cdot \bar{S}\left(K_{v}\right)_{U}\right)
$$

где $S$ - централизатор в $G$ максимального $K_{v}$-разложимого тора, $\bar{S}$ - его образ в присоединенной группе $\bar{G}=G / \mu$, а

$$
\begin{aligned}
\bar{S}\left(K_{v}\right)_{U}=\left\{s \in \bar{S}\left(K_{v}\right) \mid \chi(S) \in \mathscr{O}_{v}^{*}\right. \text { для всех } \\
\left.\qquad \text {-определенных характеров } \chi \in X^{*}(\bar{S})\right\} .
\end{aligned}
$$

Почти во всех случаях, зная абстрактное строение $\Xi_{v}$, сразу же можно указать образ группы $\xi_{v}\left(H^{1}\left(K_{v}, \mu\right)\right)$ в Aut $\Delta_{v}$, т.е. ее действие на локальной диаграмме Дынкина $\Delta_{v}$.

Чтобы описать группу $\Xi_{v}$ в общем случае, можно рассмотреть максимальное неразветвленное расширение $K_{v}^{u r}$ поля $K_{v}$ и соответствуюшую диаграмму $\Delta_{v}^{u r}$. Напомним, (см., например, [23]), что над $K_{v}^{u r}$ группа либо разложима, либо квазиразложима и можно задать каноническое вложение билдинга $\mathscr{B}$ групшы $G\left(K_{v}\right)$ в билдинг $\mathscr{B}_{1}$ группы $G\left(K_{v}^{u r}\right)$ и каноническое действие группы $\Gamma=\operatorname{Gal}\left(K_{v}^{u r} / K_{v}\right)$ на $\mathscr{B}_{1}$ такое, что образ $\mathscr{B}$ совпадает с множеством неподвижных точек в $\mathscr{B}_{1}$ относительно заданного действия группы $Г$. Кроме того, если выбрать апартамент $\mathscr{A}_{1}$ в $\mathscr{B}_{1}$, связанный с максимальньм $K_{v}^{u r}$-разложимым тором, содержащим максимальный $K_{v}$-разложимый тор, то неподвижные в $\mathscr{A}_{1}$ точки относительно $\Gamma$ образуют апартамент $\mathscr{A}$ в билдинге $\mathscr{B}$, а ограничение на $\mathscr{A}$ соответствуюших аф̆финных корней групшы $G\left(K_{v}^{u r}\right)$ задают аффинные корни группы $G\left(K_{v}\right)$. Поскольку в таблицах работы Титса [23] действие группы $\Gamma$ на $\Delta_{v}^{u r}$ задано явным образом, то это позволяет редуцировать описание $\Xi_{v}$ к разложимому и квазиразложимому случаю. Более точно, имеет место следующая коммутативная диаграмма: 


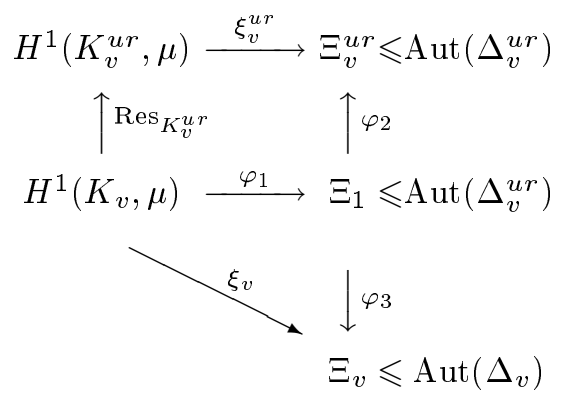

где $\varphi_{1}=\xi_{v}^{u r} \circ \operatorname{Res}_{K_{v}^{u r}}, \Xi_{1}=\operatorname{Im}\left(\xi_{v}^{u r} \circ \operatorname{Res}_{K_{v}^{u r}}\right), \varphi_{2}-$ естественное вложение, а образ $\varphi_{3}(\lambda)$ элемента $\lambda \in \Xi_{1}$ действует на $i$-ю вершину графа $\Delta_{v}$ следующим образом: надо взять произвольный аффинный корень в $\Delta_{v}^{u r}$, ограничение которого на апартамент $\mathscr{A}$ совпадает с выбранным $i$-м корнем, подействовать на него элементом $\varphi_{2}(\lambda)$, а затем взять ограничение полученного аффиннного корня на апартамент $\mathscr{A}$.

4.3. Типы $G_{2}, F_{4}, E_{8}$. Так как $\mu=1$, то $H^{1}\left(K_{v}, \mu\right)=\Xi_{v}=1$, и кроме того, для всех $v \in V_{f}$ группа $G$ разложима над $K_{v}$. Поэтому их графы Кокстера имеют соответственно вид [23]

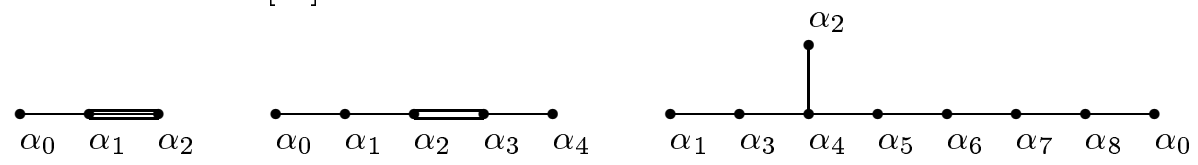

Поскольку $\Xi_{v}=1$, то максимальные типы $X_{v}$ получаются выбрасьванием произвольной вершины $\alpha_{i}$ из соответствующего графа Кокстера. В частности, для любого $v \in V_{f}$ для группы $G$ типа $G_{2}$ (соответственно, $\left.F_{4}, E_{8}\right)$ имеются три (соответственно, 5,9$)$ максимальных типа $X_{v}$. Непосредственно из определения 2.5 также следует, что любой максимальный тип $X=\left\{X_{v}\right\}_{v \in V_{f}}$ является $\mathscr{O}$-максимальным.

4.4. Тип $E_{7}: \mu=\mu_{2}, H^{1}\left(K_{v}, \mu\right) \simeq K_{v}^{*} /\left(K_{v}^{*}\right)^{2}$. Если $v \in V_{f}$, то над полем $K_{v}$ для $K_{v}$-индекса Титса имеются две возможности [21]
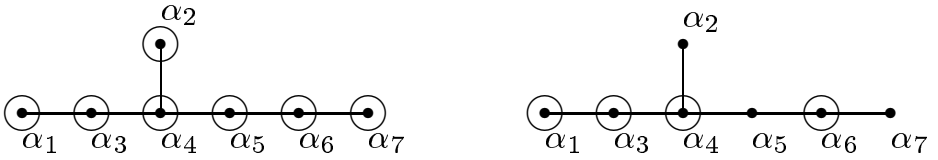

Разберем вначале первый случай. В обозначениях пункта 3.2 максимальный $K_{v}$-разложимый тор записывается в виде

$$
S=\left\langle h_{\alpha_{1}}\left(t_{1}\right) \ldots h_{\alpha_{7}}\left(t_{7}\right) \mid t_{i} \in \bar{K}_{v}^{*}\right\rangle,
$$

и центр $\mu$ порождается элементом $\alpha=h_{\alpha_{2}}(-1) h_{\alpha_{5}}(-1) h_{\alpha_{7}}(-1)$. Рассмотрим $K_{v}$-изоморфизм

$$
\lambda: S \rightarrow G_{m}{ }^{7}, \quad \prod_{i=1}^{7} h_{\alpha_{i}}\left(t_{i}\right) \mapsto\left(x_{1}, \ldots, x_{7}\right),
$$


где $x_{1}=t_{1}, x_{3}=t_{3}, x_{4}=t_{4}, x_{6}=t_{6}, x_{2}=t_{1} t_{7}, x_{5}=t_{5} t_{7}, x_{7}=t_{7}$. Так как $\lambda(\alpha)=$ $(1, \ldots, 1,-1)$, то в координатах $x_{1}, \ldots, x_{7}$ морфизму $\left.\pi\right|_{S}: S \rightarrow \bar{S}$ соответствует морфизм

$$
G_{m}^{7} \rightarrow G_{m}^{7}, \quad\left(x_{1}, \ldots, x_{7}\right) \mapsto\left(x_{1}, \ldots, x_{6}, x_{7}^{2}\right) .
$$

Тогда из (16) вытекает, что $\left|\Xi_{v}\right|=2$, причем при отождествлении $H^{1}\left(K_{v}, \mu\right)=$ $K_{v}^{*} /\left(K_{v}^{*}\right)^{2}$ единицы $\mathscr{O}_{v}^{*}$ переходят в тождественный автоморфизм графа $\Delta_{v}$, имеющего вид

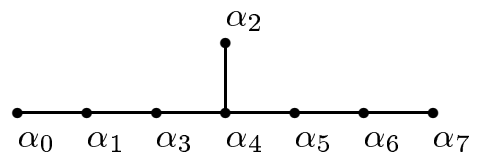

Из этой картинки следует, что $\Xi_{v}=$ Aut $\Delta_{v}$, и очевидна следующая лемма.

4.4.1. ЛЕмма. Любой максимальный тип $X_{v}$ совпадает с одним из следующих множеств:

$$
\begin{gathered}
{ }^{1} X_{v, i}=\Delta_{v} \backslash\left\{\alpha_{i}\right\}, \quad i=0,1,3,5,6,7, \\
{ }^{2} X_{v, i}= \begin{cases}\Delta_{v} \backslash\left\{\alpha_{i}\right\}, & i=2,4, \\
\Delta_{v} \backslash\left\{\alpha_{0}, \alpha_{7}\right\}, & i=0, \\
\Delta_{v} \backslash\left\{\alpha_{1}, \alpha_{6}\right\}, & i=1, \\
\Delta_{v} \backslash\left\{\alpha_{3}, \alpha_{5}\right\}, & i=3 .\end{cases}
\end{gathered}
$$

ЗАмЕчАнИЕ. Индекс слева вверху здесь и далее будет обозначать порядок стабилизатора в $\Xi_{v}$ соответствуюшего подмножества вершин графа $\Delta_{v}$.

4.4.2. ЛЕммА. Для всех чисел $i$ выполняются равенства

$$
\begin{aligned}
& H^{1}\left(K_{v}, \mu\right)_{1} X_{v, i}=\left\{x K_{v}^{* 2} \in K_{v}^{*} /\left(K_{v}^{*}\right)^{2} \mid \operatorname{ord}_{v}(x) \equiv 0(\bmod 2)\right\}, \\
& H^{1}\left(K_{v}, \mu\right)_{2} X_{v, i}=K_{v}^{*} /\left(K_{v}^{*}\right)^{2} .
\end{aligned}
$$

ДоКАЗАТЕЛЬСтво непосредственно следует из равенств $\Xi_{X_{v, i}}=1, \Xi_{2} X_{v, i}=$ Aut $\Delta_{v}$.

4.4.3. Перейдем к рассмотрению второго $K_{v}$-индекса Титса. Здесь граф Кокстера имеет вид [23]

$$
\stackrel{\alpha_{0} \quad \alpha_{1}}{\alpha_{1}} \quad \underbrace{\alpha_{2}}_{2} \quad \alpha_{3} \quad \alpha_{4}
$$

Ясно, что $\Xi_{v}=$ Aut $\Delta_{v}=1$, и любой максимальный тип $\Xi_{v}$ совпадает с одним из следуюших множеств

$$
{ }^{1} X_{v, i}=\Delta_{v} \backslash\left\{\alpha_{i}\right\}, \quad i=0, \ldots, 4,
$$

причем $H^{1}\left(K_{v}, \mu\right)_{1} X_{v, i}=K_{v}^{*} /\left(K_{v}^{*}\right)^{2}$ для всех $i$. 
4.4.4. Описание локальных максимальных типов, приведенное в 4.4.1, 4.4.3, позволяет быстро выяснить, когда максимальный тип $X=\left\{X_{v}\right\}_{v \in V_{f}}$ является $\mathscr{O}$-максимальным. Действительно, увеличение типа $X \subset X^{\prime}$ возможно лишп за счет присоединения к множеству $X_{v_{0}}$ вида $X_{v_{0}}={ }^{2} X_{v_{0}, i}, i \neq 2,4$, дополнительной вершины. При этом уменьшение подгруппы $H^{1}(K, \mu)_{X}$ происходит лишь в том случае, если найдется такой $x \in K^{*}$, что $\operatorname{ord}_{v_{0}}(x) \equiv 1(\bmod 2)$, а для остальных нормирований $v$ имеем: $x\left(K_{v}^{*}\right)^{2} \in H^{1}\left(K_{v}, \mu\right)_{X_{v}}$. Более точно, положим

$$
\begin{aligned}
& V_{1}=\left\{v \in V_{f} \mid G \text { разложима над } K_{v} \text { и } X_{v}={ }^{1} X_{v, i}\right\}, \\
& V_{2}=\left\{v \in V_{f} \mid \text { либо } G \text { неразложима над } K_{v},\right. \\
& \text { либо разложима и } \left.X_{v}={ }^{2} X_{v, i}, i=2,4\right\}, \\
& V_{3}=\left\{v \in V_{f} \mid G \text { разложима над } K_{v} \text { и } X_{v}={ }^{2} X_{v, i}, i \neq 2,4\right\}, \\
& V_{4}=\left\{v \in V_{\infty} \mid v \text { входит в описание группы } A \text { в } 3.5\right\} .
\end{aligned}
$$

Очевидно, что $V_{2}, V_{3}$ - конечные множества. Из 3.4.1 и предыдущего обсуждения вытекает, что имеет место

ПРЕДЛОЖЕНИЕ. Максимальный $\operatorname{mun} X=\left\{X_{v}\right\}_{v \in V_{f}}$ является $\mathscr{O}$-максимальным тогда и только тогда, когда для любого $v_{0} \in V_{3}$ существует $x \in K^{*}$ такой, что

a) $\operatorname{ord}_{v}(x) \equiv 0(\bmod 2)$ для всех $v \in V_{1}, \operatorname{ord}_{v_{0}}(x) \equiv 1(\bmod 2)$,

б) $x$ - положительный в $K_{v}$ әлемент для всех $v \in V_{4}$.

4.4.5. Условие а) предложения 4.4.5 эквивалентно следуюшему утверждению. Пусть $\Gamma_{K}-$ группа классов идеалов поля $K$ и для нормирования $v \in V_{f}$ через $I_{v}$ будем обозначать соответствующий элемент в $\Gamma_{K}$.

Лемма. Условие а) выполнено тогда и только тогда, когда для любого $v_{0} \in V_{3}$ имеем $I_{v_{0}} \in \Gamma_{v_{0}} \Gamma_{K}^{2}$, где $\Gamma_{v_{0}}-$ подгруппа группь $\Gamma_{K}$, порожденная $I_{v}$ для всех $v \in\left(V_{2} \cup V_{3}\right) \backslash\left\{v_{0}\right\}$.

ДоказАТЕЛЬство. Необходимость. Пусть справедливо условие а) и $x-$ соответствующий элемент. Рассмотрим разложение главного идеала $(x)$ в произведение простых. Если $(x)=\prod_{i=0}^{n} p_{i}^{n_{i}}$ и $v_{i}$ - нормирование, соответствующее простому идеалу $p_{i}$, то для всех $v_{i} \in V_{1}$ число $n_{i}$ четное и $n_{0} \equiv 1(\bmod 2)$. Переходя от равенства идеалов к равенству в группе классов идеалов, получим, что $1=I_{v_{0}}^{n_{0}} \cdot \prod_{i=1}^{n} I_{v_{i}}^{n_{i}}$ или же $I_{v_{0}} \equiv \prod_{i=1}^{n} I_{v_{i}}^{n_{i}}\left(\bmod \Gamma_{K}^{2}\right)$, что и требовалось.

Достаточность проверяется аналогично. Лемма доказана.

ЗАмечАниЕ. Так как $V_{2}, V_{3}$ - конечные множества, то утверждение леммы доставляет эффективный способ проверки свойства $\mathscr{O}$-максимальности типа $X=$ $\left\{X_{v}\right\}_{v \in V_{f}}$ для каждой конкретной групшы $G$.

4.5. Тип ${ }^{1} E_{6}: \mu=\mu_{3}, H^{1}\left(K_{v}, \mu\right) \simeq K_{v}^{*} /\left(K_{v}^{*}\right)^{3}$. Если $v \in V_{f}$, то над полем $K_{v}$ для $K_{v}$-индекса Титса имеются две возможности [21]
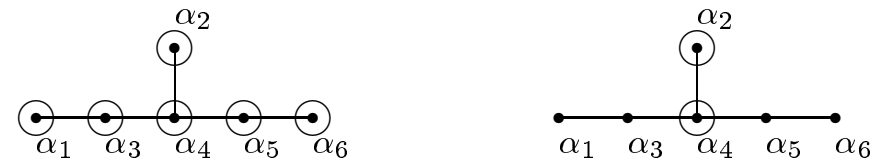
4.5.1. В разложимом случае граф Кокстера имеет вид

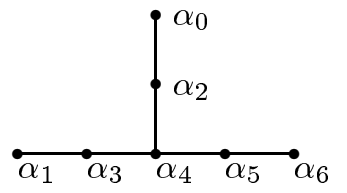

Рассуждая так же, как и в случае типа $E_{7}$, получим следующую лемму.

Лемма. 1) $\Xi_{v}=$ Aut $\Delta_{v}$ и все максимальнье типь $X_{v}$ описьваются следующими равенствами:

$$
\begin{aligned}
{ }^{1} \Xi_{v, i} & =\Delta_{v} \backslash\left\{\alpha_{i}\right\}, \quad i \neq 4, \\
{ }^{3} \Xi_{v, 4} & =\Delta_{v} \backslash\left\{\alpha_{4}\right\}, \\
{ }^{3} \Xi_{v, 0} & =\Delta_{v} \backslash\left\{\alpha_{0}, \alpha_{1}, \alpha_{6}\right\}, \\
{ }^{3} \Xi_{v, 2} & =\Delta_{v} \backslash\left\{\alpha_{2}, \alpha_{3}, \alpha_{5}\right\} .
\end{aligned}
$$

2) $H^{1}\left(K_{v}, \mu\right)_{1_{X_{v i}}}=\left\{x\left(K_{v}^{*}\right)^{3} \in K_{v}^{*} /\left(K_{v}^{*}\right)^{3} \mid \operatorname{ord}_{v}(x) \equiv 0(\bmod 3)\right\}$, $H^{1}\left(K_{v}, \mu\right)_{X_{v, i}}=H^{1}\left(K_{v}, \mu\right), i=0,2,4$.

4.5.2. В неразложимом случае граф Кокстера имеет вид

$$
\stackrel{\bullet}{\stackrel{\overline{\alpha_{1}}}{\rightleftarrows}} \alpha_{2}
$$

откуда $\Xi_{v}=$ Aut $\Delta_{v}=1$. Поэтому все максимальные типы совпадают с ${ }^{1} X_{v, i}=$ $\Delta_{v} \backslash\left\{\alpha_{i}\right\}, i=0,1,2$, и $H^{1}\left(K_{v}, \mu\right)_{1_{v, i}}=H^{1}\left(K_{v}, \mu\right)$.

4.5.3. Пусть $X=\left\{X_{v}\right\}_{v \in V_{f}}$ - максимальный тип. Положим

$V_{1}=\left\{v \in V_{f} \mid G\right.$ разложима над $K_{v}$ и $\left.X_{v}={ }^{1} X_{v, i}\right\}$,

$V_{2}=\left\{v \in V_{f} \mid\right.$ либо $G$ неразложима над $K_{v}$, либо разложима и $\left.X_{v}={ }^{3} X_{v, 4}\right\}$,

$V_{3}=\left\{v \in V_{f} \mid G\right.$ разложима над $K_{v}$ и $\left.X_{v}={ }^{3} X_{v, i}, i=0,2\right\}$.

Суммируя приведенную выше информацию с учетом 3.4.1 имеем следующее

ПРЕДЛОЖЕНИЕ. Максимальный тип $X=\left\{X_{v}\right\}_{v \in V_{f}}$ является $\mathscr{O}$-максимальным тогда и только тогда, когда для любого $v_{0} \in V_{3}$ существует $x \in K^{*}$ такой, что $\operatorname{ord}_{v}(x) \equiv 0(\bmod 3)$ для всех $v \in V_{1} u \operatorname{ord}_{v_{0}}(x) \not \equiv 0(\bmod 3)$.

СлеДСТВИЕ. Максимальный mun $X=\left\{X_{v}\right\}_{v \in V_{f}}$ является О-максимальнымм тогда и только тогда, когда для любого $v_{0} \in V_{3}$ имеем $I_{v_{0}} \in \Gamma_{v_{0}} \Gamma_{K}^{3}$, где $\Gamma_{v_{0}}-$ nодгруппа группь $\Gamma_{K}$, порожсденая $I_{v}$ для всех $v \in\left(V_{2} \cup V_{3}\right) \backslash\left\{v_{0}\right\}$.

4.6. Тип ${ }^{2} E_{6}$. Если $v \in V_{f}$ и $G$ над $K_{v}$ имеет внешний тип, то граф̆ Кокстера имеет вид [23]

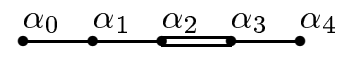

Отсюда следует, что $\Xi_{v}=$ Aut $\Delta_{v}=1$, и поэтому максимальные типы $X_{v}$ задаются равенствами

$$
{ }^{1} X_{v, i}=\Delta_{v} \backslash\left\{\alpha_{i}\right\}, \quad i=0, \ldots, 4,
$$


и $H^{1}\left(K_{v}, \mu\right)_{X_{v, i}}=H^{1}\left(K_{v}, \mu\right)$.

Перейдем к описанию $\mathscr{O}$-максимальных типов. Пусть $X=\left\{X_{v}\right\}_{v \in v_{f}}$ - максимальный тип. Положим

$V_{1}=\left\{v \in V_{f} \mid G\right.$ разложима над $K_{v}$ и тип $X_{v}$ совпадает с одним

$$
\text { из множеств } \left.{ }^{1} X_{v, i} \text { из (18) }\right\} \text {, }
$$

$V_{2}=\left\{v \in V_{f} \mid\right.$ либо $G$ неразложимая над $K_{v}$ группа внутреннего типа и

$X_{v}$ - любое, либо $G$ разложима и $X_{v}$ совпадает с множеством ${ }^{3} X_{v, 4}$ из (18) $\}$,

$V_{3}=\left\{v \in V_{f} \mid G\right.$ разложима над $K_{v}$ и тип $X_{v}$ совпадает с одним

$$
\text { из множеств }{ }^{3} X_{v, i}, i \neq 4 \text {, из (18)\}. }
$$

Пусть $L$ - квадратичное расширение поля $K$, над которым $G$ имеет внутренний тип. Напомним, что

$$
H^{1}(K, \mu)=\operatorname{Ker}\left(L^{*} /\left(L^{*}\right)^{3} \rightarrow K^{*} /\left(K^{*}\right)^{3}\right) .
$$

Очевидно, что если $L \cdot K_{v} \neq K_{v}$, то нормирование $v$ поля $K$ имеет единственное продолжение на $L$, которое будем обозначать той же буквой. Поэтому если $l\left(L^{*}\right)^{3} \in H^{1}(K, \mu)$, то для таких нормирований $v$ справедливо равенство $v(l) \equiv 0(\bmod 3)$. Если же $L \cdot K_{v}=K_{v}$, то $v$ имеет два продолжения $v^{1}, v^{2}$ на $L$, и тогда $v^{1}(l)+v^{2}(l) \equiv 0(\bmod 3)$.

ПрЕДЛОЖЕНИЕ. Максимальный тип $X=\left\{X_{v}\right\}_{v \in V_{f}}$ является $\mathscr{O}$-максимальныцм тогда и только тогда, когда для любого $v_{0} \in V_{3}$ существует $x \in L^{*}$ такой, что

$$
\begin{aligned}
& \operatorname{ord}_{v^{i}}(x) \equiv 0(\bmod 3), \quad i=1,2, \text { для всех } v \in V_{1} u \\
& \operatorname{ord}_{v_{0}^{1}}(x) \equiv 1(\bmod 3), \quad \operatorname{ord}_{v_{0}^{2}}(x) \equiv 2(\bmod 3) .
\end{aligned}
$$

ДокАЗАТЕЛЬСТво. Из описания локальных групп $H^{1}\left(K_{v}, \mu\right)_{X_{v}}$ следует, что $H^{1}(K, \mu)_{X}=\left\{l\left(L^{*}\right)^{3} \in H^{1}(K, \mu) \mid \operatorname{ord}_{v^{i}}(l) \equiv 0(\bmod 3), i=1,2\right.$, для всех $\left.v \in V_{1}\right\}$.

Поскольку тип $X$ можно увеличить лишь за счет компонент $v \in V_{3}$, то уменьшение $H^{1}(K, \mu)_{X}$ происходит лишь тогда, когда для любого $v_{0} \in V_{3}$ найдется $l\left(L^{*}\right)^{3} \in H^{1}(K, \mu)_{X}$ такой, что $v_{0}^{1}(l) \not \equiv 0(\bmod 3)$. Тем самым, остается только установить, что из сушествования элемента $l \in L^{*}$ с указанными в утверждении предложения свойствами следует существование $\widetilde{l}\left(L^{*}\right)^{3} \in H^{1}(K, \mu)$ с аналогичными свойствами. Пусть $1 \neq \sigma \in \operatorname{Gal}(L / K)$. Тогда для всех $a \in L v_{0}^{2}(a)=v_{0}^{1}(\sigma(a))$. Рассмотрим элемент $\widetilde{l}=\sigma(l) / l$. Очевидно, что $\widetilde{l}\left(L^{*}\right)^{3} \in H^{1}(K, \mu)_{X}$ и, кроме того,

$$
v_{0}^{1}(\widetilde{l})=v_{0}^{1}(\sigma(l))-v_{0}^{1}(l)=v_{0}^{2}(l)-v_{0}^{1}(l) \equiv 1(\bmod 3),
$$

что и требовалось доказать.

СлеДСТвиЕ. Максимальныц ти $X=\left\{X_{v}\right\}_{v \in V_{f}}$ является $\mathscr{O}$-максимальнвцм тогда и только тогда, когда для любого $v \in V_{3}$ имеем $I_{v_{0}^{1}} I_{v_{0}^{2}}^{2} \in \Gamma_{v_{0}} \Gamma_{L}^{3}$, где $\Gamma_{v_{0}}-$ подгруппа группь $\Gamma_{L}$, порожденная $I_{v^{i}}, i=1,2$, для всех $v \in\left(V_{2} \cup V_{3}\right) \backslash\left\{v_{0}\right\}$.

Для доказательства следует повторить рассуждение из леммы 4.4.5. 


\section{§5. Классификация МАП групп типов $B_{n}, C_{n}$}

5.1. Типы $B_{n}, C_{n}$ рассматриваются аналогично типам $E_{7},{ }^{1} E_{6}$. Поэтому приведем лишь окончательный ответ без дополнительных пояснений.

Тип $B_{n}$. Для $K_{v}$-индекса Титса имеются две возможности [21]

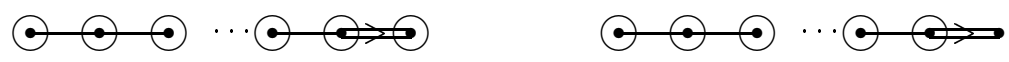

Локальные диаграммы Дынкина имеют соответственно вид

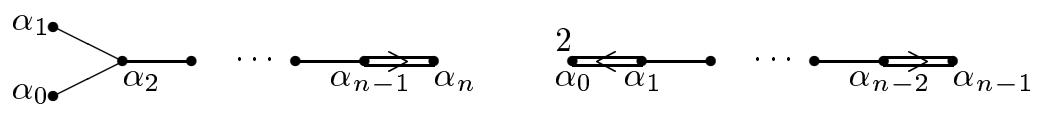

В первом случае $\left|\Xi_{v}\right|=\mid$ Aut $\Delta_{v} \mid=2$, и поэтому максимальные типы $X_{v}$ совпадают с

$$
\begin{aligned}
& { }^{1} X_{v, i}=\Delta_{v} \backslash\left\{\alpha_{i}\right\}, \quad i=0,1, \\
& { }^{2} X_{v, i}= \begin{cases}\Delta_{v} \backslash\left\{\alpha_{i}\right\}, & i=2, \ldots, n, \\
\Delta_{v} \backslash\left\{\alpha_{0}, \alpha_{1}\right\}, & i=0 .\end{cases}
\end{aligned}
$$

Очевидно также, что для ${ }^{j} X_{v, i}$ справедливы равенства

$$
\begin{aligned}
& H^{1}(K, \mu)_{2} X_{v, i}=K_{v}^{*} /\left(K_{v}^{*}\right)^{2}, \\
& H^{1}(K, \mu)_{{ }_{1} X_{v, i}}=\left\{x\left(K_{v}^{*}\right)^{2} \in K_{v}^{*} /\left(K_{v}^{*}\right)^{2} \mid \operatorname{ord}_{v}(x) \equiv 0(\bmod 2)\right\} .
\end{aligned}
$$

Во втором случае $\Xi_{v}=$ Aut $\Delta_{v}=1$, откуда любой максимальный тип $X_{v}$ совпадает с одним из следующих:

$$
{ }^{1} X_{v, i}=\Delta_{v} \backslash\left\{\alpha_{i}\right\}, \quad i=0, \ldots, n-1,
$$

причем $H^{1}(K, \mu)_{1} X_{v, i}=K_{v}^{*} /\left(K_{v}^{*}\right)^{2}$.

Пусть $X=\left\{X_{v}\right\}_{v \in V_{f}}$ - максимальный тип. Положим

$$
\begin{aligned}
& V_{1}=\left\{v \in V_{f} \mid G \text { разложима над } K_{v} \text { и } X_{v}={ }^{1} X_{v, i}\right\}, \\
& V_{2}=\left\{v \in V_{f} \mid \text { либо } G \text { неразложима над } K_{v}, \text { либо } G\right. \text { разложима и }\left.X_{v}={ }^{2} X_{v, i}, i=2, \ldots, n\right\}, \\
& V_{3}=\left\{v \in V_{f} \mid G \text { разложима над } K_{v} \text { и } X_{v}={ }^{2} X_{v, 0}\right\}, \\
& V_{4}=\left\{v \in V_{\infty} \mid v \text { входит в описание групшы } A \text { в } 3.8\right\} .
\end{aligned}
$$

ПрЕДЛОЖенИЕ. Максимальный тип $X=\left\{X_{v}\right\}_{v \in V_{f}}$ является $\mathscr{O}$-максимальныцм тогда и только тогда, когда для любого $v \in V_{3}$ существует такой $x \in K^{*}$, что

a) $\operatorname{ord}_{v}(x) \equiv 0(\bmod 2)$ для всех $v \in V_{1}$, $\operatorname{ord}_{v_{0}}(x) \equiv 1(\bmod 2)$,

б) $x$ - положительный әлемент в $K_{v}$ для всех $v \in V_{4}$.

Условие а) можно сформулировать в следуюшем виде. 
Лемма. Условие а) выполняется тогда и только тогда, когда $I_{v_{0}} \in \Gamma_{v_{0}} \Gamma_{K}^{2}$ для любого $v_{0} \in V_{3}$, где $\Gamma_{v_{0}}-$ подгруппа группь $\Gamma_{K}$, порожденная $I_{v}$ для всех $v \in\left(V_{2} \cup V_{3}\right) \backslash\left\{v_{0}\right\}$.

5.2. Тип $C_{n}$. Для $K_{v}$-индекса Титса имеются три возможности

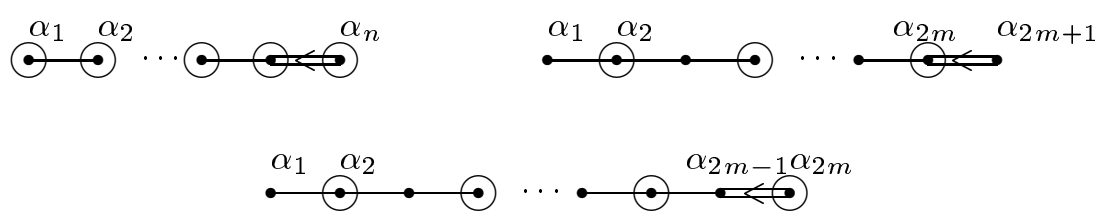

Во втором случае $n=2 m+1$ - нечетное число, а в третьем $n=2 m$-четное. Локальные диаграммы Дынкина имеют соответственно вид

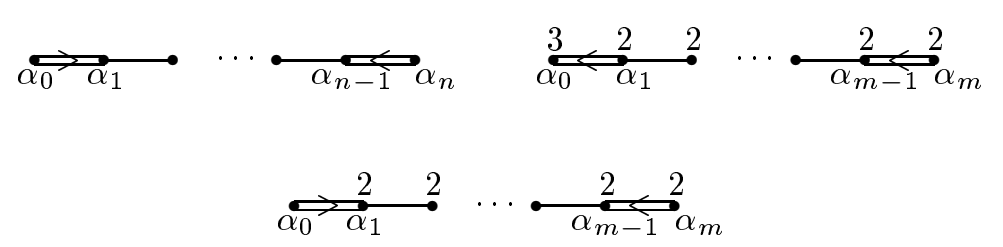

если $m>1$. Если же $m=1$, то во втором и третьем случаях локальная диаграмма Дынкина имеет вид

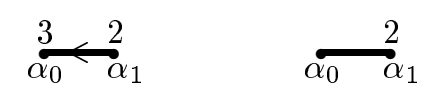

Во всех случаях, кроме $K_{v}$-разложимого, $\Xi_{v}=$ Aut $\Delta_{v}=1$, поэтому максимальные типы $X_{v}$ задаются равенствами

$$
{ }^{1} X_{v, i}=\Delta_{v} \backslash\left\{\alpha_{i}\right\}
$$

причем $H^{1}(K, \mu)_{1} X_{v, i}=K_{v}^{*} /\left(K_{v}^{*}\right)^{2}$.

В $K_{v}$-разложимом случае $\Xi_{v}=$ Aut $\Delta_{v}$, откуда для максимальных типов $X_{v}$ сушествуют следуюшие возможности:

$$
\begin{aligned}
{ }^{1} X_{v, i} & =\Delta_{v} \backslash\left\{\alpha_{i}\right\}, \quad i=0, \ldots, n \quad\left(i \neq\left[\frac{n}{2}\right]+1, \text { если } n \text { нечетное }\right), \\
{ }^{2} X_{v, i} & =\Delta_{v} \backslash\left\{\alpha_{i}, \alpha_{n-i}\right\}, \quad i=0, \ldots,\left[\frac{n}{2}\right], \\
{ }^{2} X_{v,\left[\frac{n}{2}\right]+1} & =\Delta_{v} \backslash\left\{\alpha_{\left[\frac{n}{2}\right]+1}\right\}, \quad \text { если } n \text { нечетное. }
\end{aligned}
$$

Пусть $X=\left\{X_{v}\right\}_{v \in V_{f}}$ - максимальный тип. Положим

$$
\begin{gathered}
V_{1}=\left\{v \in V_{f} \mid G \text { разложима над } K_{v} \text { и } X_{v}={ }^{1} X_{v, i}\right\}, \\
V_{2}=\left\{v \in V_{f} \mid \text { либо } G \text { неразложима над } K_{v}, \text { либо } G\right. \text { разложима и } \\
\left.\qquad X_{v}={ }^{2} X_{v,\left[\frac{n}{2}\right]+1}, \text { если } n \text { нечетное }\right\}, \\
V_{3}=\left\{v \in V_{f} \mid G \text { разложима над } K_{v} \text { и } X_{v}={ }^{2} X_{v, i}, i=0, \ldots,\left[\frac{n}{2}\right]\right\}, \\
V_{4}=\left\{v \in V_{\infty} \mid v \text { входит в описание группш } A \text { в } 3.9\right\} .
\end{gathered}
$$


ПРЕДЛОЖЕНИЕ. Максимальный тип $X=\left\{X_{v}\right\}_{v \in V_{f}}$ является $\mathscr{O}$-максимальным тогда и только тогда, когда для любого $v_{0} \in V_{3}$, существует такой $x \in K^{*}$, чmо

a) $\operatorname{ord}_{v}(x) \equiv 0(\bmod 2)$ для всеx $v \in V_{1}$, $\operatorname{ord}_{v_{0}}(x) \equiv 1(\bmod 2)$,

б) $x$ - положительный әлемент в $K_{v}$ для всех $v \in V_{4}$.

ЛЕмма. Условие а) выполняется тогда и только тогда, когда $I_{v_{0}} \in$ $\Gamma_{v_{0}} \Gamma_{K}^{2}$ для любого $v_{0} \in V_{3}$, где $\Gamma_{v_{0}}-$ подгруппа группь $\Gamma_{K}$, порожденная $I_{v}$ для всеx $v \in\left(V_{2} \cup V_{3}\right) \backslash\left\{v_{0}\right\}$.

\section{Список литературы}

1. Бондаренко $A . A$. К классификации максимальных арифметических подгрупп в ортогональных группах типа $D_{l} / /$ Докл. АН БССР. 1974. Т. 18. № 9. С. 773-776.

2. Бондаренко $A$. A. К проблеме максимальности арифметических подгрупп в ортогональных группах типа $B_{n} / /$ Матем. заметки. 1974. Т. 16. №1. С. 151-161.

3. Бондаренко A. А. Классификация максимальных арифметических подгрупп ортогональных групп типа $D_{l} / /$ Докл. АН БССР. 1975. Т. 19. № 11. С. 969-972.

4. Бондаренко $A$. A. K классификации максимальных арифметических подгрупп в разложимых группах // Матем. сб. 1977. Т. 102. № 2. С. 155-172.

5. Бондаренко $A . A$. Классификация максимальных ариффметических подгрупп неопределенных ортогоналњных групп типа $B_{l} / /$ Матем. сб. 1985. Т. 127. № 1. С. 72-91.

6. Бондаренко A. A. О максимальности арифметических подгрупп неопределенных ортогональных групп типа $D_{l} / /$ Матем. сб. 1990. Т. 181. №3. С. 388-401.

7. Бондаренко A. A. Классификация максимальных арифметических подгрупп неопределенных ортогональных групп типа $C_{l} / /$ Докл. АН Беларуси. 1994. Т. 38. № 3. С. 29-32.

8. Платонов В.П. К проблеме максимальности арифметических групп // Докл. АН CCCP. 1971. T. 200. №3. С. 530-533.

9. Платонов В. П. Арифометическая теория алгебраических групп // УМН. 1982. Т. 37. №3. C. $3-54$.

10. Bruhat F., Tits J. Groupes reductifs sur un corps local // Publ. Math. I. H. E. S. 1972. V. 41. P. 5-252.

11. Bruhat F., Tits J. Groupes reductifs sur un corps local. Chap. II. Schemas en groupes. Existence d'une doneé radicielle valueé // Publ. Math. I. H. E. S. 1984. V. 60. P. 5-184.

12. Платонов В. П., Рапинчук A. С. Алгебраические группы и теория чисел. М.: Наука, 1991.

13. Rohlfs $J$. Die maximalen arithmetische definierten Untergruppen zerfallender einfacher Gruppen // Math. Ann. 1979. V. 244. P. 219-231.

14. Margulis G.A., Rohlfs J. On the proportionality of covolumes of discrete subgroups // Math. Ann. 1986. V. 275. P. 197-205.

15. Allan N.D. The problem of the maximality of arithmetic groups // Proc. Symp. Pure Math. 1966. V. 9. P. 104-109.

16. Allan N. D. On the maximality of $\operatorname{Sp}(L)$ in $\operatorname{Sp}_{n}(K) / /$ Rev. Colomb. Math. 1970. V. 4 . № 1. P. 7-15.

17. Allan N. D. On the commensurability class of the Siegel modular group // Bull. Amer. Math. Soc. 1968. V. 74. № 1. P. 114-118.

18. Borel A. Density and maximality of arithmetic subgroups // J. Reine Angew. Math. 1966. V. 224. P. $78-89$.

19. Бурбаки Н. Группы и алгебры Ли. Гл IV-VI. М.: Мир, 1972.

20. Рагунатан М. С. Дискретные подгрупшы групп Ли. М.: Мир, 1977. 
21. Титс Ж. Классификация полупростых алгебраических групп // Математика. 1968. T. 12. № 2. C. 110-143.

22. Стейнберг Р. Лекции о группах Шевалле. М.: Мир, 1975.

23. Tits J. Reductive groups over local fields // Proc. Symp. Pure Math. 1979. V. 33. P. 29-69.

Институт математики АН Беларуси, Минск

Поступила в редакцию

30.12 .1996 